\title{
NEUE ORPHEUSVERSE AUF DEM DERVENI-PAPYRUS
}

The Papyrus found in 1962 contains a commentary on an Orphic Theogony including the quotations of more than two dozen epic verses of "Orpheus", several of them being already known as such, whilst others are new. Amongst these are five verses known hitherto only from «Homer": two of them were from the very beginning acknowledged as "Orpheus", the resting ones being considered as quotations from Homer, against the Commentator's assertion. In the following paper it will be demonstrated that these verses, too, are "Orpheus". The clue to the problem's solution: The Last Homeric Poet is a

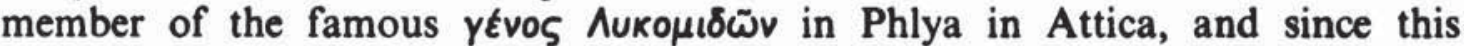
Genos had of old a genuine Orpheus-Tradition, there are more traces of this kind of poetry in the Last Poet's components in "Homer" than is generally realized.

Bei jedem neuen Papyrusfund muss man mit neuen Erkenntnissen rechnen, die bisheriges Wissen entweder bestätigen oder in Frage stellen. Nichts Geringeres als die These von der «orphischen Plagiatliteratur" aus Homer und Hesiod ist diesmal zur Diskussion gestellt. Denn der im Jahre 1962 gefundene Derveni-Papyrus (nachfolgend D.P.) hat einige neue Orpheusverse gebracht, die in epischen Fragen - also insbesondere zum Problem der Interrelation zwischen Orpheus und Homer wie auch zwischen Orpheus und Hesiod - zur Revision bisheriger Positionen zwingen. Aus den zahlreichen Dichtungszitaten, die der Papyrus bringt (es sind deren etwa zwei Dutzend), erfordern in diesem $\mathrm{Zu}$ sammenhang die folgenden unsere Aufmerksamkeit:

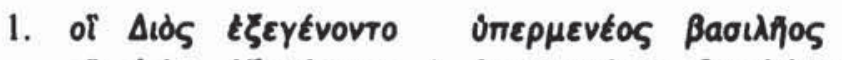

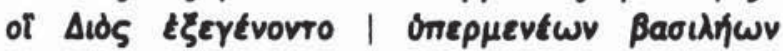

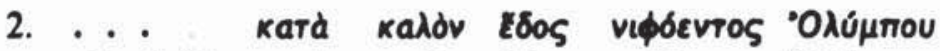

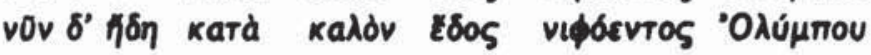

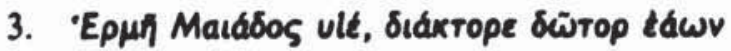

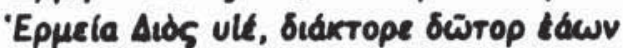

D.P. Col. 4,2

Il. V $637+$ VIII 236

D.P. Col. 8,2

Hy. XV 7

D.P. Col. 22,4

Od. VIII 335 


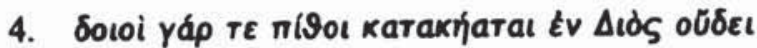

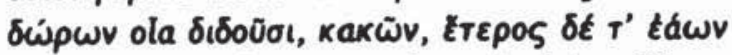

D.P. Col. 22,6 f.

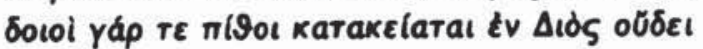

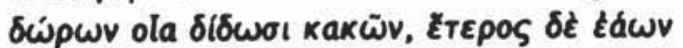

Il. XXIV $527 \mathrm{f}$.

Diese fünf Verse werden auf dem Derveni-Papyrus als Orpheusverse zitiert, während sie vor dem Fund des Papyrus nur aus Homer bekannt waren. Wie ist die Herkunft der Verse nun zu beurteilen?

Inhalt des Papyrus ist ein Prosa-Traktat, in dem "Orpheus»-Dichtung interpretiert wird, und zwar höchst eigenwillig und so gut wie immer falsch: was aber für das Nachfolgende, in dem es um die Interrelation zwischen «Orpheus» und «Homer» geht, ganz ausser Betracht bleiben kann '. Im erhaltenen Teil des Traktats werden fast zwei Dutzend Orpheusverse angeführt, und als solche Zitationen findet man in der vierten, achten und letzten der erhaltenen Columnen auch die fünf hier zu betrachtenden Verse. Nimmt man sie wie alle andern beigezogenen poetischen Zitate als "Orpheus»-Dichtung, so vermehrt sich der Bestand an Versen mit doppeltem Heimatrecht - bei Orpheus und Homer - ganz beträchtlich ${ }^{2}$. Andernfalls müsste man annehmen, dass sie innerhalb des Traktats ganz oder teilweise eine Ausnahme bilden, dass sie nämlich von dessen Verfasser aus dem Homer beigezogen sind, ohne dass er ihren Dichter mit Namen genannt hätte. Im Abdruck des Papyrus in der Zeitschrift für Papyrologie und Epigraphik ${ }^{3}$ kommt eine offenbar zwiespältige Auffassung zum Ausdruck, da die im Traktat sich findenden Zitationen von "Orpheus»-Dichtung stets unterstrichen sind

' Nach dem Fund des Papyrus im Februar 1962 hat Kapsoménos in verschiedenen Zss. einen ersten kurzen Bericht gegeben. Die dann erschienenen weiteren Beiträge anderer Forscher brauchen hier nicht aufgeführt zu werden, da sie sich alle mit der geistesgeschichtlichen Einordnung der dem Papyrus zu entnehmenden "orphischen" Lehren, also nicht mit dem im Nachfolgenden zu behandelnden Problem befassen. Vom Orpheusproblem als einem epischen Problem werden nachstehend von den Publikationen des Vf. die folgenden abgekürzt zitiert:

Orpheus: Orpheus, Der Sänger und seine Zeit, Bern und München 1970.

Sänger d. Vz.: Der Sänger der Vorzeit, Drei Kapitel zur Orpheusfrage, Bern und München 1980.

Peisistratos: Peisistratos und sein homerischer Dichter, Ein Kapitel Prolegomena ad Homerum, Bern und München 1983.

Verk. Muse: Die Verkannte Muse, Dichtersprache und geistige Tradition des Parmenides, Bern 1986.

"Alpha 1»: "Homer Alpha 1", Zs. emerita 54, 1986, $202 \mathrm{ff}$., bes. $218 \mathrm{ff}$.

2 s. zunächst den sehr unvollständigen (s.u.) Index $\mathrm{V}$ in Kern Orphic. Frgm. (1922), S. 398.

${ }^{3}$ ZPE 47, 1982, Anhang mit eigener Paginierung nach S. 300. Es handelt sich wohl um eine provisorische Herausgabe. Vgl. schon ZPE 1, 1967, $31 \mathrm{f}$. Der Vf. dieser Blätter verdankt ein Exemplar der Freundlichkeit Reinhold Merkelbachs. 
- darunter auch die hier zu betrachtenden Verse der vierten und achten Columne -, diejenigen der letzten Columne hingegen nicht, in ihnen also offenbar Homer-Zitationen zu sehen sind. Auch der Autor eines beachtenswerten Beitrags zu diesem neuen Papyrus, Walter Burkert ${ }^{4}$, ist offensichtlich der gleichen Auffassung, da er die Zitationen der vierten und achten Columne zwar als Orpheusverse nimmt, bei denen der 22. Columne aber zunächst unpersönlich übersetzt «Klar ist dies in den folgenden Versen..." und bei deren erstem in Klammer "Od. VIII 335» hinzusetzt, und er ebenso auch fortfährt «Klar ist es auch in folgendem...», wobei er in Klammer "Il. XXIV 527 f.» hinzufügt. Durch beides, die unpersönliche Ausdrucksweise - anstatt wörtlich «er macht klar" - sowie die Beifügung der Homerstellen, ist deutlich, dass auch er in den drei Versen der letzten Columne nicht Orpheus $=$ sondern Homerzitationen sieht ${ }^{5}$. Und mancher andere dürfte wohl der gleichen Auffassung sein, ungeachtet der Inkonzinnität gegenüber den Parallelfällen in der vierten und achten Columne. Wie steht es also um diese Verse: sind sie "Orpheus» oder "Homer"?

Vorweg ist zu bedenken: der Zweck der Zitation lässt die Annahme zweier Homer-Zitate kaum zu. Der Autor des Traktats will ja die Be-

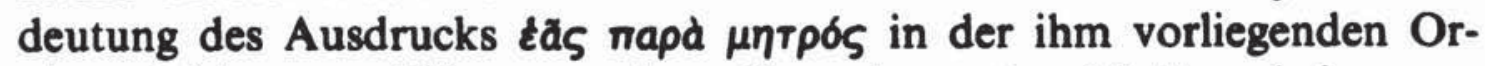
pheus-Dichtung erklären - dass dies "der guten Mutter» heisse wozu er als Beleg dafür, dass Orpheus dies meine, nichts anderes als zwei Verse aus dem Homer - aus Od. VIII und aus Il. XXIV - beizubringen gewusst hätte, während er eine solche Auffassung doch gerade aus Orpheus hätte belegen müssen: schon das ist überaus fragwürdig.

Aber auch der Wortlaut steht einer solchen Annahme entgegen. Mit einer selbstverständlichen Bestimmtheit werden die Verse als OrpheusVerse eingeführt: «'Er' offenbart es aber auch in folgenden Versen, dass

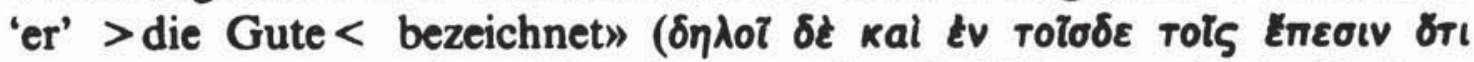

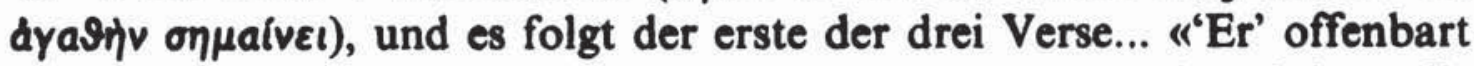

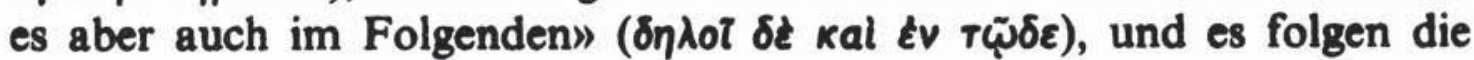
beiden andern Verse. Das dreimalige in den Verben enthaltene Subjekt 'er' kann stets nur Orpheus meinen. Nicht nur weil der ganze Traktat ausschliesslich Paraphrase und Erklärung von "Orpheus»-Dichtung ist, und auch die angenommene Bedeutung des Ausdrucks bei "Orpheus» ja doch nur aus diesem selbst belegt werden kann: diese Ausdrucksweise ist im ganzen Traktat konstant die übliche Einführung aller «Orpheus»$93 \mathrm{ff}$.

4W. Burkert, "Orpheus und die Vorsokratiker», Antike und Abendland 14, 1968,

3 So übrigens gleich von Anfang an bereits Kapsoménos in seinen provisorischen Mitteilungen (oben A. 1), s. z.B. Gnomon 35, 1963, 222 f. 
Zitationen, d.h. für das "er offenbart» und «er bezeichnet» gibt es genügend andere Vorkommensfälle, in denen monoton stets nur Orpheus gemeint und auf dessen Dichtung verwiesen ist. So zunächst die folgenden zu ס̄

Col. $4,3 \quad \ldots$ है folgt,

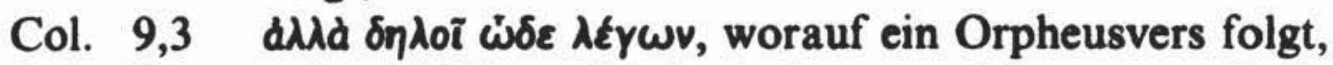

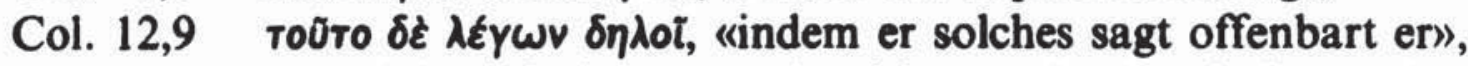
was sich auf einen gerade zitierten Orpheushalbvers bezieht,

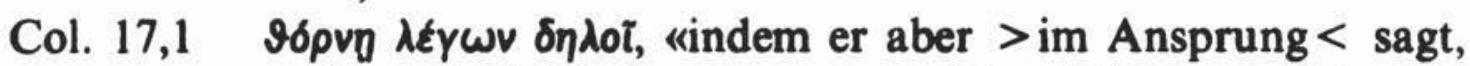
meint (zeigt/offenbart) er", was sich wiederum auf Aussagen in der Orpheusdichtung bezieht.

Auch ein $\varepsilon \delta \eta \lambda \omega \sigma \varepsilon v$ in Col. 7,11 kann nur Orpheus meinen. Und für

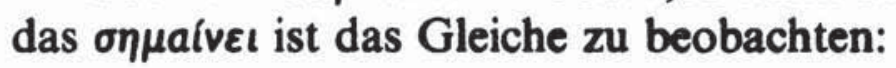

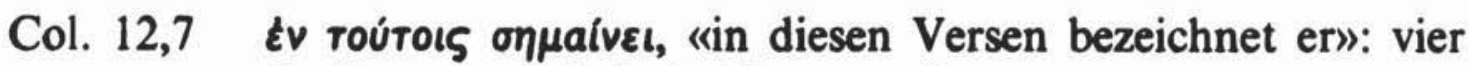
Orpheusverse sind als Zitat gerade vorangegangen,

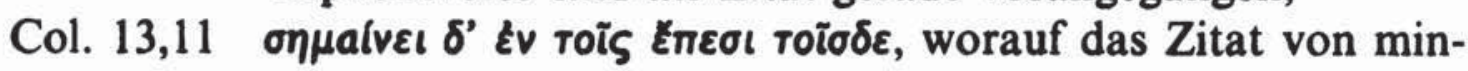
destens Einem Orpheusvers folgt,

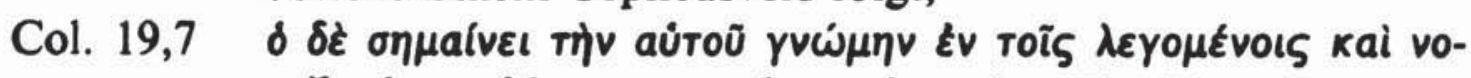
$\mu \iota \zeta o \mu \varepsilon v 0 ı \zeta$ pr $\mu a \sigma$, «er aber zeigt seine Einsicht mittels der gewöhnlichen Wörter der Umgangssprache an" ${ }^{6}$, und auch diese Äusserung meint nach dem Vorangegangenen selbstverständlich Orpheus.

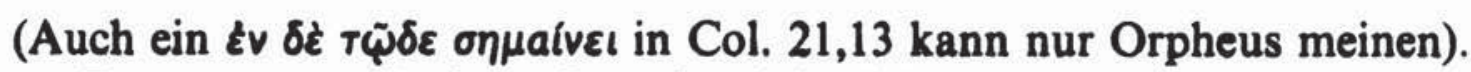

Dem ganzen Traktat liegt ja sicherlich eine zusammenhängende Dichtung zu Grunde ?, aus der einige Verse schon lange als Orpheusverse bekannt waren ${ }^{8}$, und als Verfasser dieser Dichtung und Künder ihrer Lehren wird ja auch Orpheus zweimal ausdrücklich benannt (Col. 14,2 und 6). Subjekt ist Orpheus auch an den zahlreichen andern Stellen, wo auf - vorangehend oder nachfolgend - zitierte Orpheusdichtung ver-

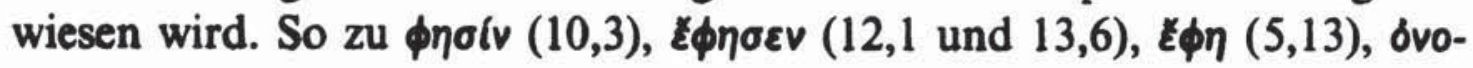

- So übersetzt W. Burkert, a.O., 95.

7 So auch schon W. Burkert, a.O., 96.

8 Beispiele: Col. $3,8=$ Orph. fr. $245,1=247,1=-334$, vgl. Plat. Symp. 218 B. - Col. $12,4 \mathrm{ff} .=$ Orph. fr. $67 \mathrm{~b}$. - Col. $13,12=$ Orph. fr. $21 \mathrm{a}, 2=168,2$, vgl.
Plat. Leg. IV $715 \mathrm{E}$. 


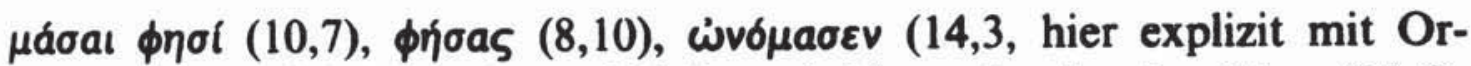

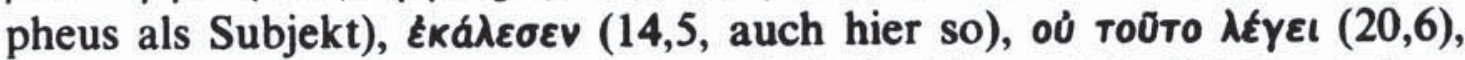

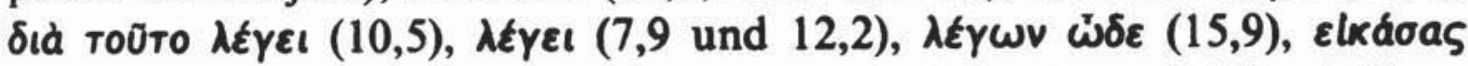

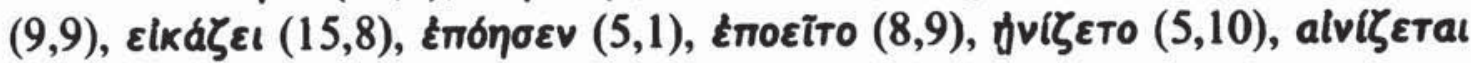

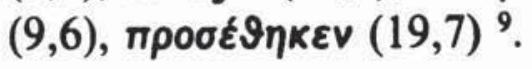

Der Häufigkeit und Üblichkeit solcher auf Orpheus weisenden Ausdrucksweise stehen nur sehr wenige Fälle mit einem andern Subjekt gegenüber, über das aber niemals ein Zweifel aufkommen kann: $\lambda \varepsilon y \varepsilon \iota$ in Col. 11,7 meint "To हैпоร", "der zitierte Vers besagt", wobei dieser na-

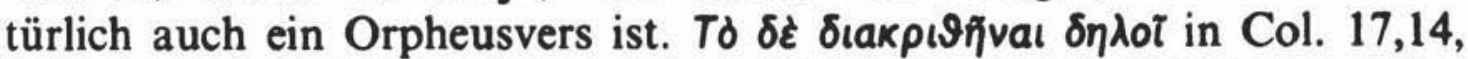

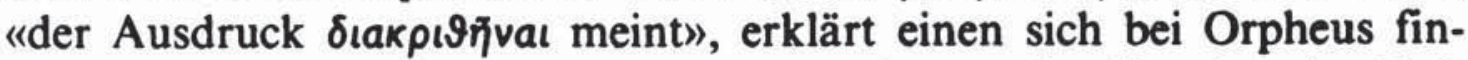

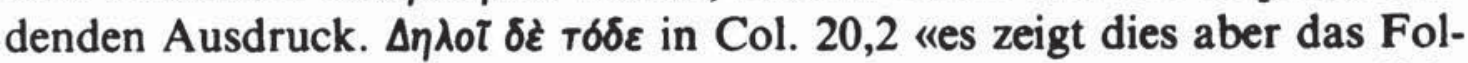
gende» weist auf den folgenden Vers, einen Orpheusvers natürlich.

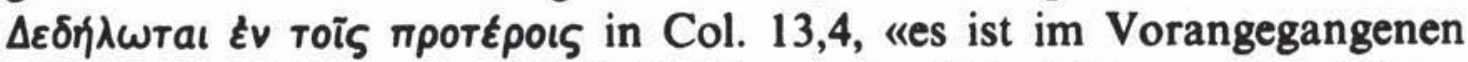
erklärt», und so auch einfach $\delta \varepsilon \delta \dot{1} \lambda \omega t a$ in Col. 12,1 "es ist erklärt», womit gleichfalls «im Vorangegangenen" gemeint ist.

Ein anderer Dichter als Subjekt ist bei solcher durchgehend uniformer und eindeutiger Ausdrucksweise ausgeschlossen. Vom klaren Text "Er offenbart» in der Übersetzung durch Änderung ins Unpersönliche abzuweichen ist nicht statthaft, es sei denn man ginge von der unzulässigen - weil sachlich unzutreffenden - Voraussetzung aus, dass was im Homer steht nicht auch bei Orpheus gestanden haben könne (bezw. "dürfe»), obwohl ein Blick auf den Index «Epici ab Orphicis adhibiti» in Kerns Orphicorum Fragmenta rasch darüber belehrt, dass es genug Beispiele von "Homer-Versen" gibt, die sich auch in "Orpheus-Dichtung» finden ${ }^{10}$. Und die Zitationen der letzten Columne anders zu nehmen als die der vierten und achten geht auch nicht an. Dass dem Verfasser des Traktats die in Frage stehenden Verse aus dem Homer bekannt gewesen sein sollten, könnte man zudem nur dann annehmen, wenn man ihm ein bedenkliches Vorgehen d.h. eine wissentliche Fälschung unterstellen wollte. Gibt es aber Gründe ihm eine solche anzulasten?

Wer heute noch der Lobeck-Kernschen Theorie folgt, ist mit seinem Urteil freilich rasch zur Hand: alle "Orpheus»-Dichtung ist «bekanntlich» Falsifikation («Plagiat») aus Homer und Hesiod, also «beweisen» die vorliegenden Zitationen "homerischer» Verse als "Orpheus»-Dichtung das Gleiche: sie sind weitere Beispiele für das Vorgehen der wor-

\footnotetext{
9 Alles Vorstehende unter Ausschluss unsicherer Ergänzungen.
}

10 Dabei ist dieser Index keineswegs vollständig, s. weiter unten. 
phischen Fälscher» d.h. deren Dreistigkeit, mit der sie «notorische» Homerverse ganz ungeniert für Orpheus ausgeben ".

Wer aber Bedenken hat derartiges als Behauptung ohne Beweis einfach zu statuieren, wird aus der Sache selbst urteilen. Er wird sich die Homer-Stellen kritisch besehen und feststellen:

Der Hermesvers ist fraglos eine Götterprädikation, eine dem Kult entstammende Epiklese. Nun war nicht Homer wohl aber Orpheus zu allen Zeiten als Schöpfer und Patron religiöser Dichtung bekannt, während es andererseits schwer vorstellbar ist, dass die Griechen ihre rituellen Götteranrufungen der frivolen Unterhaltungsdichtung entnommen haben sollten - der Zusammenhang in Od. VIII, der Demodokos-Gesang von Ares und Aphrodite, dürfte ja hinreichend bekannt sein.

Es kommt hinzu: In Od. VIII ist Hermes als Sohn des Zeus, im Orpheusvers dagegen als Sohn der Maias gepriesen. Der «orphische Fäl-

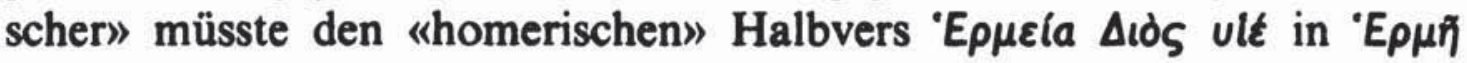
Maı́óos viḱ abgeändert haben. Da der Verfasser des Traktats den Vers ja nur um des ihn beschliessenden Worts záwv willen zitiert, ist aber kein Grund einzusehen, weshalb er sich zu dieser unnötigen Änderung der ersten Vershälfte entschlossen haben sollte. Man müsste darum annehmen, dass nicht der Autor des Traktats sondern ein früherer worphischer Fälscher» den Vers zwecks Übernahme in seine "Orpheus»Dichtung abgeändert habe. Das hiesse dann zweierlei: zunächst dass der Verfasser des Traktats den Vers eben als "Orpheus»-Vers vorgefunden habe, zugleich aber auch, dass der frühere Fälscher sich für Hermes als Maíóos ulós, als er den Vers dem Od. VIII entnahm, auf Od. XIV 435

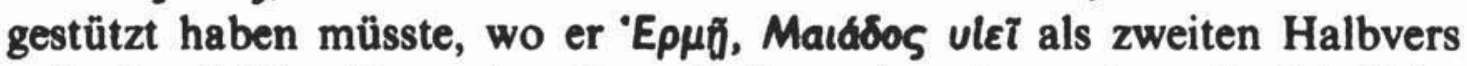
gefunden hätte. Zur ersten fragwürdigen Annahme, dass die feierliche

"Repräsentative Ausserungen dazu s. Orpheus 20,3. Nach Otto Kern ist zwar - entgegen seinem eigenen Index "Epici ab Orphicis adhibiti" - «Homer als Vorbild der Orphiker - abgesehen natürlich von der epischen Sprache - völlig auszuscheiden", Rel. d. Gr. II $28 \mathrm{f}$. (wobei man freilich annehmen müsste, dass die "epische Sprache" - wie die hier zu behandelnden Verse zeigen - auch das Inhaltliche zwangsläufig mit einschliesst: die Prädikation des Hermes, das Mythologem von den beidon Fässern, usw.). Aber ungeachtet eines solchen (unwirksam gebliebenen) Satzes bei Kern und ungeachtet auch einer einzelnen brieflichen Äusserung an den Vf. - "die Rederei von den Fälschern und Plagiatoren beruht auf irrigen Denkkategorien des 19. Jahrhundertsw (R. Merkelbach) - erweist sich die LobeckKernsche Theorie noch in der zweiten Hälfte des 20. Jhdts. als virulent, wenn z.B. N.J. Richardson versichert, dass Homers Demeterhymnus «was taken over and apparently adapted as an Orphic poem» (The Homeric Hymn to Demeter, Oxford 1974, S. 85, vgl. 169, 234, wozu Th. Gelzer, Mus. Helv. 34, 1977, 249), und wenn man sieht, dass noch in der jüngsten wissenschaftlichen Literatur ein irgend geartetes Abrücken von Lobeck-Kern geradezu unvorstellbar ist (s. z.B. Verk. Muse 142 ff., auch Sänger d. Vz. 87,2). 
Hermes-Epiklese just in des Demodokos Schwank originär sei, würde damit gleich noch eine zweite hinzugefügt, da ein solches Zusammenstücken disparater Parallelen - zumal bei dem hier vorliegenden Positionswechsel - kaum denkbar ist ${ }^{12}$. In der mit dem Namen Orpheus signierten religiösen Tradition hingegen dürfte das prädizierende Maı́$\delta \circ \varsigma$ viદ viel eher ursprünglich sein, weil nämlich einerseits Homer und die Homertradition niemanden nach der mütterlichen Abkunft bezeich-

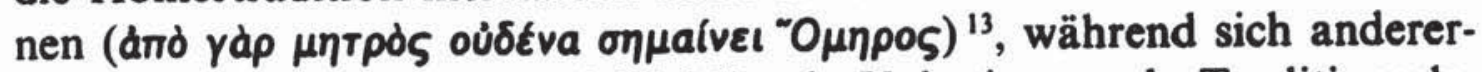
seits das Maláóos ulk - gleichfalls als Vokativ - als Traditionselement auch im "orphischen» Hymnus auf Hermes noch findet (Orph. Hy. 28,1$)^{14}$, nachdem es aus der homerisch-hesiodeischen Konkordanz - Od. XIV $435=$ Hes. fr. 217,2 - nach dem «Fundamentalschluss» ohnehin als ein seit ältester Zeit der Orpheustradition angehörendes Element anzusprechen ist ${ }^{15}$.

Und weiter: Innerhalb der Komik der Situation ist die Anrede Apollons an Hermes mit sakraler Formel extrem inadäquat, ja deplaziert, denn es ist Missbrauch kultischer Form, also Blasphemie. «Die Häufung der feierlichen Epitheta in der Anrede an den Bruder dient hier der komischen Wirkung ${ }^{16}$. Sicherlich: die Frage ist nur, ob der Dichter dieser Partie diesen Vers eigens in der Absicht, diese komische Wirkung zu erzielen, so feierlich gestaltet d.h. ob er ihn geschaffen hat. Und diese unumgängliche Frage ist entschieden zu verneinen.

Der Dichter der überaus seltsamen $O d$. VIII-Partie hat den Vers offenkundig gerade nicht geschaffen sondern be nutzt: er war ihm nämlich aus älterer Tradition geläufig, wie die Parallelen zeigen. Zu dem

12 Dies auch für Hoekstra nicht, Mnemosyne 10, 1957, 139 ff., Orpheus 38,2. Aber das Undenkbare anzunehmen erübrigt sich: eine andere Erklärung stellt sich nämlich ein, wenn man beachtet, dass Od. XIV $435=$ Hes. fr. 217,2, wozu gleich.

${ }_{13}$ Apollonios Lex. Hom. s. Moגlove. In der Orpheustradition hingegen gibt es das and $\mu \eta \tau \rho \delta$ s, s. bald.

is Die orphischen Hymnen «können hier unbedenklich beigezogen werden, wo sie trotz später Entstehung .... an alte orphische Uberlieferung ... anknüpfen»", W. Theiler, Unters. z. ant. Lit., Berlin 1970, S. 24, A. 28. Von der besonders deutlich zu Tage tretenden Kontinuität auf dem Gebiet des Kultisch-Religiösen über Jahrhunderte hinweg spricht - aus anderem Anlass - auch A. Heubeck, Glotta 39, 1960, 164 f. Zur Illustration dieser Kontinuität gerade in Orphicis diene auch die wörtliche Übereinstimmung der Petrusapokalypse mit den bei Pindar, Platon, u.a., sich findenden "orphischen" Aussagen, die bereits von A. Dieterich (Nekyia, Leipzig und Berlin 19132) hervorgekehrt ist (Vf. Orpheus 154-157, 343).

is Der «Fundamentalschluss»: unter dieser Bezeichnung zunächst eingefuhrt $\mathrm{Or}$ pheus 34-38 (u.d.F.), dann neu gefasst Sänger d. Vz. 64-71. Ein bemerkenswertes Beispiel: Peisistratos 19 f. (Zur Problematik der induktiven Schlussfolgerung s. unten).

${ }_{16}$ So Ameis-Hentze, Hom. Od. erkl. I 2 (1922), S. 49. 


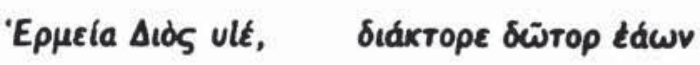

\begin{tabular}{|c|c|c|c|}
\hline treten: & $\begin{array}{c}\text {... Maı́dos } u l k, \\
\ldots \\
\ldots\end{array}$ & 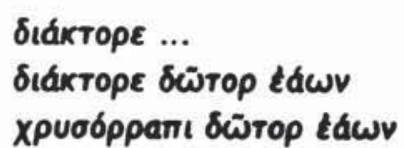 & $\begin{array}{l}\text { Hy. Herm. } 514 \\
\text { Hy. XVIII } 12 \\
\text { Hy. XXIX } 8\end{array}$ \\
\hline & v & 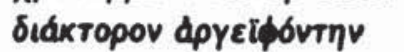 & Od. I 84 und H.E. $68{ }^{17}$ \\
\hline
\end{tabular}

Der Hermeshymnus mit seinem Maı́dos ulê folgt nicht dem Od. VIII: von einer Benutzung der Odyssee durch einen jüngeren Hymnendichter kann also auch hier nicht die Rede sein ${ }^{18}$ : vielmehr liegt gemeinsames Traditionsgut vor. Und die beiden zuletzt genannten Parallelen in Od. I einer = und im Hesiod andererseits sind aufschlussreich: nach dem bereits mehrfach erprobten "Fundamentalschluss» d.h. jener Konkordanzenregel, wonach in den homerisch-hesiodeischen Parallelen altes mythisch-poetisches Gut der präliterarischen Orpheustradition vor-

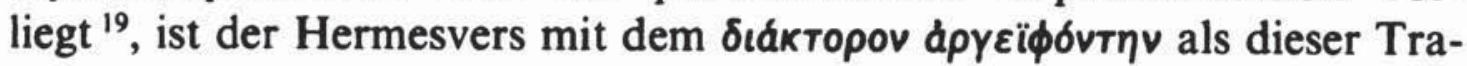
dition zugehörig anzusehen. Daher überrascht es nicht, gerade das Element

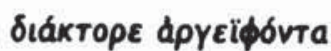

- wiederum - auch im orphischen Hymnus in gleicher Position anzutreffen (Orph. Hy. 28,3). Gleiches ist ja gerade für das ( $E \rho \mu \tilde{)})$ Maı́óos

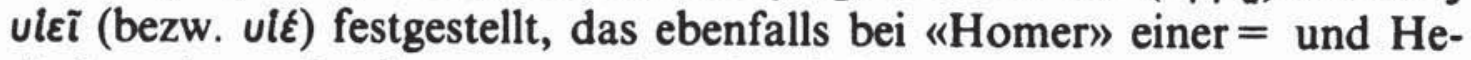
siod andererseits begegnet, also ebenfalls der Orpheustradition entstammt und sich darum ebenfalls, und zwar auch in gleicher Position, im orphischen Hymnus findet $(28,1)^{20}$.

Das $\delta \tilde{\omega}$ тор $\varepsilon \dot{c} \omega v$ (in Od. VIII 335) hat zwar keine Hesiodparallele, sicherlich nur zufällig, weil dieser alte Gott Hermes dem ganz auf Zeus eingeschworenen Askräer nicht viel bedeutet ${ }^{21}$. Aber einerseits ist dies

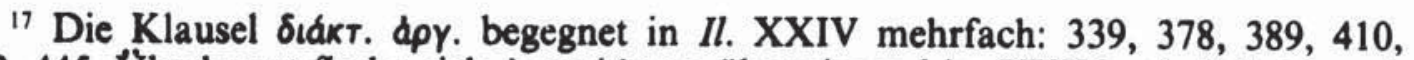

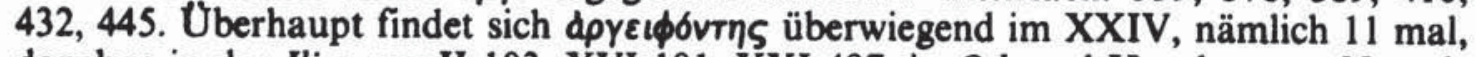
daneben in der Ilias nur II 103, XVI 181, XXI 497, in Od. und Hy. dagegen 29 mal.

${ }^{18}$ Das ist für die "homerischen" Hymnen schon gezeigt: Peisistratos 41-60. Im

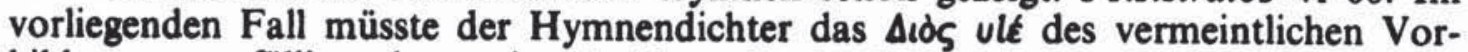
bildverses "zufällig" ebenso in Maıdós vlé abgeändert haben wie der "orphische Fälscher».

${ }^{19}$ s. oben Anm. 15.

${ }^{20}$ Dazu Sänger d. Vz. a.O., bes. S. 67 («Zweitens»), wozu Peisistratos a.O., oben, Anm. 14.

${ }^{21}$ Hermes wird in der Theogonie unter anderen Zeuskindern in nur zwei Zeilen erwähnt, Th. 938 f., daneben das zufällige - rein konventionell-formelhafte - Vorkommen in Th. 444 und Erga 68 (und nicht anders steht es in den Fragmenten): die Nennung dieses alten Gottes geht nie über poetisehe Konvention hinaus. Zu Hesiods einseitiger Festlegung auf Zeus s. Mark D. Northrup, TAPhA 110, 1980, $223 \mathrm{ff}$., Verk. Muse 132-137. 


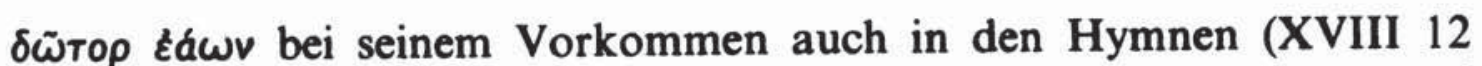
und XXIX 8) ebenfalls als überkommene Wendung gleicher Art - und darum wohl auch gleicher Provenienz - ausgewiesen. Und andererseits hat das vom $O d$. VIII-Dichter kurz zuvor gebrauchte parallele und

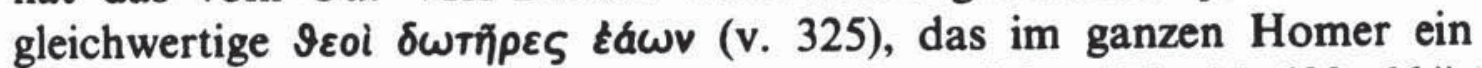
Hapax ist, durchaus die erwarteten Hesiodparallelen (Th. 46, 633, 664), wobei das dreifache Vorkommen die auch dem Hesiod bereits tradi erte Wendung ausweist, sodass die nach der Konkordanzenregel gewiesene Herkunft aus der orpheischen Muse abermals keinem Zweifel unterliegen kann. (Dies übrigens umso weniger, als ja dieser Fall beim

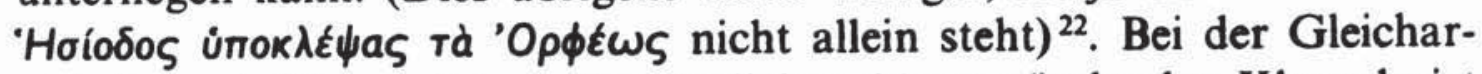
tigkeit der beiden auf dem altertümlichen źáwv gründenden Klauseln ist sicher, dass auch das zu Hermes gehörige Traditionselement $\delta \tilde{\omega}$ тор źáwv (das den Od. VIII-Vers mit zwei der Hymnen verbindet) wie die andern der Orpheustradition entstammt. Damit ist also der ganze Hermesvers dieser Provenienz.

Im Blick auf die ermittelte Herkunft dieses mythisch-poetischen Elements ist zu dessen erstem Halbvers noch anzufügen, dass die im Traktat zitierte Orpheusversion

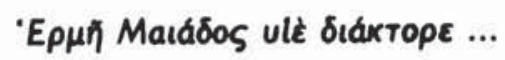

als tradiert in dem

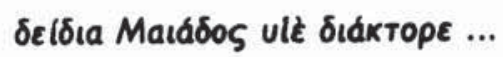

des Hymnus eine Parallele und Bestätigung hat (v. 514, vgl. auch die

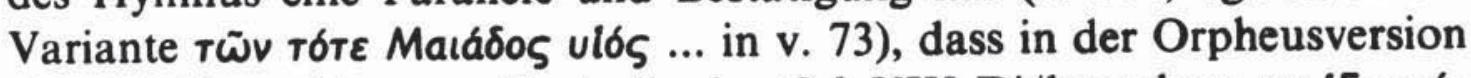
also die ältere Form vorliegt, die der Od. VIII-Dichter dann zu 'Ep $\mu \varepsilon i a$

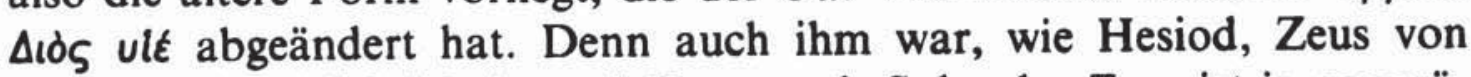
überragender Wichtigkeit: und Hermes als Sohn des Zeus ist ja gegenüber seiner mütterlichen Abkunft fraglos sekundär ${ }^{23}$.

${ }_{22} \mathrm{Zu}$ des Hesiod «Hypoklepsie» s. Orpheus passim (bes. 49,5), sodann Sänger d. Vz. $16 \mathrm{ff}$. (wo bereits ein von Mazon bereitgestellter Musterfall aufgegriffen ist), dazu 26-29, 64-72, Peisistratos $30 \mathrm{ff}$, Verk. Muse $142 \mathrm{f}$.

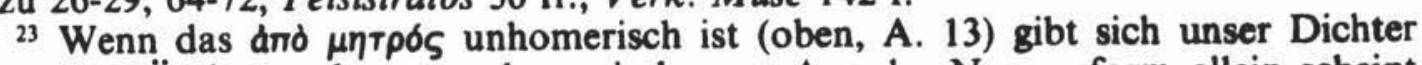
mit seiner Änderung bewusst «homerisch». - Aus der Namensform allein scheint

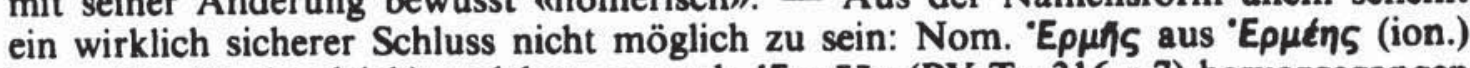

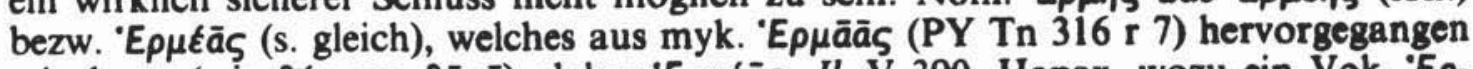

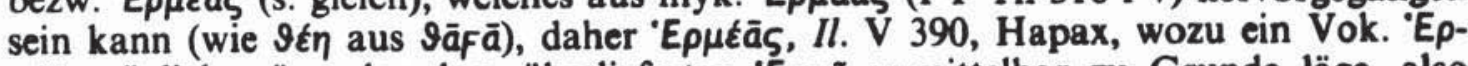

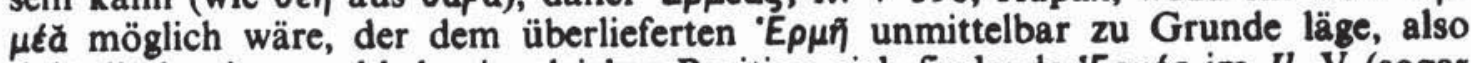
daktylisch wie sowohl das in gleicher Position sich findende 'Epuḱ im Il. V (sogar im Dat.) als auch das Substitut $\delta \varepsilon\left(\delta \_a\right.$ im Hymnus. Hingegen: «La forme épique 'Ep- 
Ist nun diese Erklärung richtig, dass der sub judice stehende Vers der in Od. VIII als Homervers, auf dem Derveni-Papyrus als Orpheusvers erscheint - der präliterarischen Orpheustradition entstammt, so folgt daraus, dass er in irgendwelcher dem Autor des Traktats geläufigen Orpheusdichtung zu Recht seinen Platz hat - von "Orphikerfälschung" also keine Rede sein kann -, dass der Vers vielmehr legitim herangezogen werden konnte, um die Bedeutung des $\varepsilon_{a} a_{S}$ bei Orpheus darzutun ${ }^{24}$... ebenso aber auch, dass dem «homerischen» Od. VIIIDichter dieser Vers aus der Tradition der or pheis chen Muse geläufig gewesen sein muss. Wie hat man das zu verstehen?

Nun: die betreffende Partie des Od. VIII, zumindest des Demodokos merkwürdiger Sang von Ares und Aphrodite ${ }^{25}$, ist ja nach der Homeranalyse Werk des Letzten Dichters, jenes Dichters also, der aus andern Zusammenhängen inzwischen als L y k o mide erwiesen ist, d.h. als Angehöriger des berühmten attischen Seher $=$ und Priestergeschlechts, das in Phlya seinen Stammsitz und sein Gentilheiligtum hatte. Und dies Geschlecht war - nach später Bezeugung, die sich jedoch verifizieren lässt - aus seiner Herkunft vom berühmten Neleischen Pylos Träger einer genuinen musisch-poetischen Orpheustradition ${ }^{26}$. Aus dieser seiner gentilizischen Muse hat der Dichter bei der Gestaltung («Redaktion») der beiden homerischen Grossepen allenthalben auch poetisches Gut einfliessen lassen, weshalb es neben den garnicht zu übersehenden Anleihen aus Homer eine gleichfalls nicht zu übersehende Anzahl von Orpheusversen und überhaupt mythisch-poetischen Elementen der Orpheustradition im «Homer» gibt (Erscheinungen, die sich natürlich nur

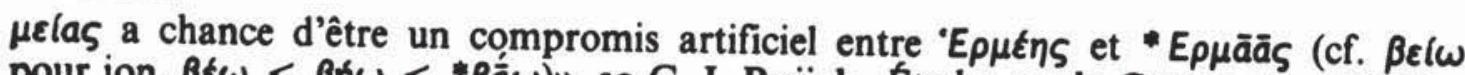
pour ion. $\beta \hat{\varepsilon} \omega<\beta \hat{j} \omega$ < $\beta \bar{a} \omega) »$, so C. J. Ruijgh, Etudes sur la Grammaire et le Vocabulaire du Grec Mycénien, Amsterdam 1967, p. 80,57, 218,87, 266, n. 154. Auch Schwyzer erklärt "hom." 'Eppelas aus 'Eppéas, Gr. Gram. I 562 Zusatz 5. Vgl. auch Chantraine, Dict. Et. s.v., Helmut Rix, Hist. Gram. d. Gr., Darmstadt 1976, 140.

${ }_{24}$ Der Kommentator kannte selbstverständlich auch andere Orpheus-Dichtung als die, welche er im Traktat erklärt: an einer Stelle belegt er ja beispielsweise, dass

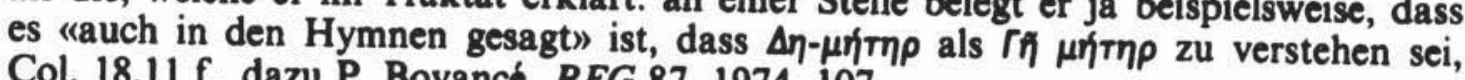
Col. 18,11 f., dazu P. Boyancé, REG 87, 1974, 107.

${ }^{25}$ Dessen Kernstück hat bei der antiken Kritik sogar Anstoss erregt: 333-342 Ev

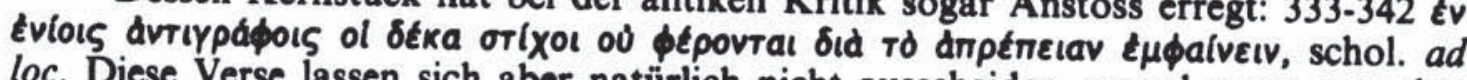
loc. Diese Verse lassen sich aber natürlich nicht ausscheiden, zumal wenn man den Hintergrund dieses «Prothalamion» beachtet, wofür hier nur auf E.A.S. Butterworth, Some Traces etc. $125 \mathrm{ff}$. verwiesen sei. Bei Von der Mühll findet man auch Sonst Beispiele dafür, dass "B»-Partien oft so bedenklich sind, dass schon antike Kritik durch Athetesen glaubte Remedur schaffen $Z$ : Können.

${ }^{26}$ Der Letzte Dichter, "B» der Analyse (Bethe, Von der Mühll, Merkelbach), als Lykomide: diesen Nachweis bringt der Peisistrâtos. Eine Kurzfassung der Argumentation Verk. Muse 24-29. Die Verifikation des spät Bezeugten: Peisistratos $61 \mathrm{ff}$. Zur historischen Bedeutung des Lykomiden auch Verk. Muse $155 \mathrm{f}$. 
auf die der Hand dieses Letzten Dichters entstammenden Partien beschränken) ${ }^{27}$. Das sind zunächst alle jene Verse, die man früher für "Orphikerplagiate» aus Homer gehalten hatte ${ }^{28}$, dazu aber auch noch andere, von denen einige bereits erkannt und besprochen sind ${ }^{29} . \mathrm{Zu}$ ihnen tritt jetzt ja auch der auf dem Derveni-Papyrus in Col. 8 zitierte Orpheusvers

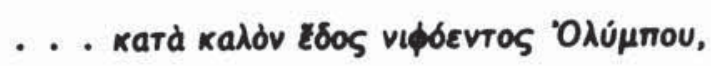

der bisher ebenfalls nur als «Homer»-Vers bekannt war:

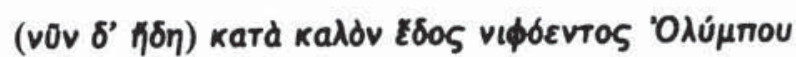

(Hy. XV 7) und der als alttradiertes Element der orpheischen Muse noch in den "orphischen» Hymnen zweimal eine Entsprechung hat:

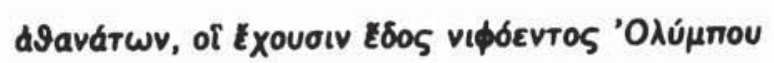

(Orph. Hy. 25,7 nebst Variante in 59,12). Dieser Vers ist nun zwar in seiner auf dem Derveni-Papyrus zitierten Form von den eingangs genannten Autoren als Orpheusvers unmittelbar anerkannt, jedoch ohne dass auf sein Verhältnis zum «homerischen» Hymnus XV eingegangen wäre. Und gerade dies ist ja doch wiederum höchst aufschlussreich, denn im Hymnus handelt es sich um ein zusammengehöriges Verspaar

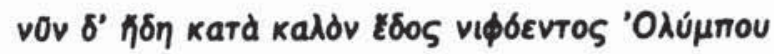

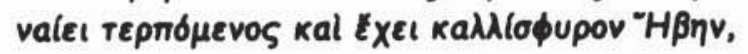

${ }^{27}$ Sänger d. Vz. 10-31, Peisistratos 19 f., 29-38, 42 ff., 76-79, emerita 54, 1986, $218 \mathrm{ff}$.

${ }_{28}$ Kern, Orphic. Frgm. Index V. Daselbst fehlen aber Il. V 499-501 = Orph. fr. 268 (eine der umfangreichsten und bedeutendsten Úbereinstimmungen zwischen "Homer" und "Orpheus"), sowie Il. VIII $5=$ XIX $101(\sim$ VIII 20) $=$ Orph. fr. $167 b, 5$, ferner Hy. Dem. $418-423=$ Orph. fr. $49,21-27$, Hy. Dem. 8-12 = Orph. fr. 49,63-68, Hy. Dem. 17 f. $+33-36=$ Orph. fr. 49,69-75, Hy. Dem. 248 f. $=$ Orph. fr. $49,92-94$, Hy. Dem. $255-262=$ Orph. fr. $49,95-100$, Hy. Dem. 54 f. $=$ Orph. fr. 49,102-105.

${ }_{29}$ Ausser den in voriger Anm. genannten Stellen (von denen Od. XI 427 mehrfach besprochen ist) sind noch zu nennen: Od. X 86 (gleichfalls mehrfach), XI 213, 386 , Il. VIII 12-16, 46, 48, 398, 423, 483, 539, XIV 201, 302, 246, II 551, Od. VII $83,94,101$, Hy. Dem. 2 f., 5,6 f., 31 f., 70, 80, 90, 305 f., 334-337, 338 f., 349 ff., 409 f., wozu Il. XV 121 f. mitsamt Il. I 1, Hy. Aphr. 56-71, 117-121, 196 ff., 214, 221,240 , u.a.m., s. in den jeweiligen Indices. 
dessen zweiter Vers auf jenen berühmt-berüchtigten Nekyia-Passus führt:

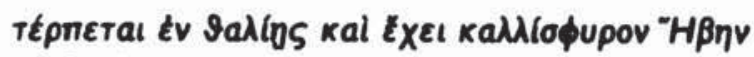

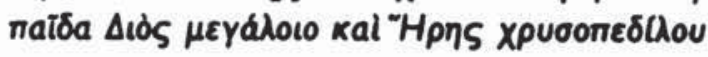

(Od. XI 603 f.), wobei einerseits die Hesiodparallelen (Hy. XV 7b Hes. Th. 953b, Od. XI $604=$ Hes. Th. 952 und fr. 25,28 f. bezw. 229,8 f., auch $O d$. XI 602a = Hes. fr. 23,21) nach dem "Fundamentalschluss» auf Orpheus führen ${ }^{30}$, und andererseits die Verse Od. XI 602-604

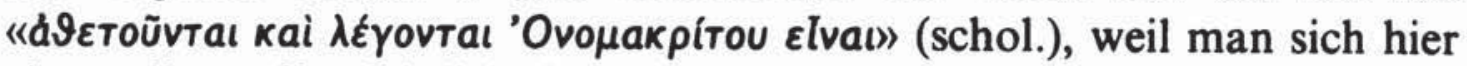
eben allzu offensichtlich in einem unhomerischen Kontext befindet: nämlich in Dichtung des Lykomiden, des Letzten Dichters, mit dessen altererbter Orpheustradition ${ }^{31}$.

Und gleichartig liegen die Dinge ja auch für die beiden Orpheus-

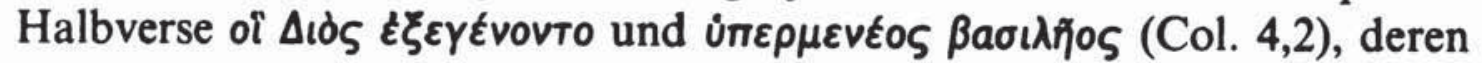
erster zunächst zwei Parallelen beim Lykomiden hat, nämlich die wörtliche in $I l . \mathrm{V} 637^{32}$ und daneben die Variante of $\varepsilon \vartheta \varepsilon v \varepsilon \xi \varepsilon \gamma \varepsilon v 0 v T 0$ (wobei das $\varepsilon \vartheta \varepsilon v$ nicht nur metrisches sondern auch bedeutungsmässiges Äqui-

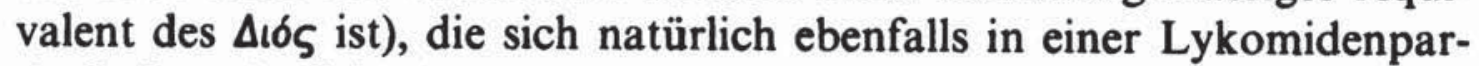
tie findet, nämlich $\mathrm{Il}$. XX 305, und hier auch wieder im Zusammenhang mit einer ohnehin längst schon als Orpheusvers bekannten poetischen Fügung: $I l$. XX 308 (= Orph. fr. 4) ${ }^{33}$. Der zweite Halbvers hat seine -.Parallele in Il. VIII 236, also ebenfalls in einer Partie des Lykomiden, in der schon einige Orpheuselemente hervorgetreten sind (s. auch gleich).

Wer in der Identifikation des Letzten Dichters zu folgen vermag, wird nicht überrascht sein, die in Frage stehenden "Homer»-Verse den des Hy. XV wie auch Il. V 637a und VIII 236 b - jetzt auf dem Papyrus auch als "Orpheus»-Dichtung zitiert zu finden. Und in diesen ganzen genannten ansehnlichen Kreis von Orpheus-Dichtung im «Homer» (und zwar nicht zufällig stets in den Partien des Letzten Dichters)

${ }^{30}$ So auch kurz danach Od. XI $612=$ Hes. Th. 228, wozu Orpheus $40 \mathrm{f}$.

${ }^{31}$ Die Verse Od. XI 602-604 lassen sich natürlich nicht entfernen, sie sind vom Dichter des grösseren Zusammenhangs der Nekyia (vgl. schol. ad 568, Wilamowitz, Hom. Unt. 140 ff. zu Od. XI 566-631), der mit 602-604 die der Heraklessage ins Gesicht schlagende Erfindung entschuldigt, Von der Mühll, Philologus 93, 1938, 9 und $R E$ Suppl. 7,727. - Der lykomidische Letzte Dichter ist so gut wie sicher mit Onomakritos identisch: Peisistratos $87 \mathrm{ff}$.

${ }^{32}$ Il. V $637=$ «B», Von der Mühll, Hypomnema $98+100 \mathrm{f}$.

${ }^{33}$ Zu Il. XX 307 f. Hy. Aphr. 196 ff.: s.' Von der Mühll, Hyp. 303 (mit. A. 33), Peisistratos 46 f. - NB: Welche Gleichartigkeit: Hy. XV 7 dem Od. XI 604 verbunden wie $I l$. XX 305 dem XX 308. 
gehört nun also auch der Hermes-Vers in Od. VIII, der ebenfalls in eine "Orpheus»-Dichtung Eingang gefunden hat, die - geschaffen aus der präliterarischen Orpheustradition - dem Autor des Traktats vorgelegen hat.

Auch dies wird noch deutlicher, wenn man sieht, dass auch er im Sang des Demodokos keineswegs allein steht, dass auch er in einem entsprechend aufschlussreichen Kontext begegnet. Betrachten wir darum kurz auch noch die vier zugehörigen Verse 338-341:

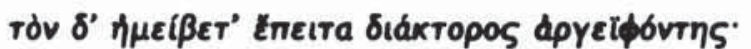

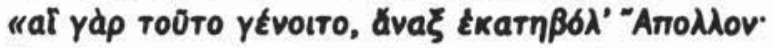

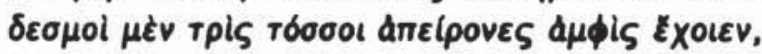

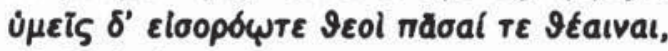
340

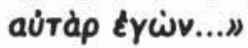

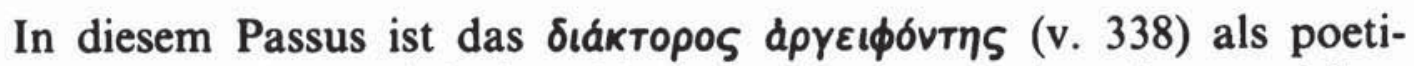
sches Element der orpheischen Muse bereits besprochen. Im anschlies-

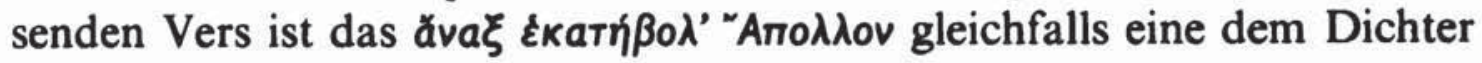
tradierte Fügung. Das zeigen zunächst seine Varianten in den Versen 334 und 323, und alle diese drei Formen haben noch Parallelen ausserhalb der Odyssee:

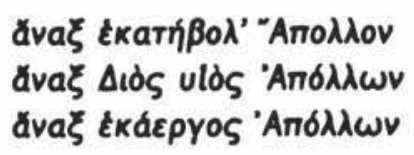

$$
\begin{aligned}
339= & \text { Hy. Ap. } 140 \\
334= & \text { Hes. fr. } 235,1 \\
323= & \text { Hy. Ap. } 357,382,420,440, \\
& \text { Hy. Herm. 222, 500 }
\end{aligned}
$$

Für die Hymnen hat man den gleichen Letzten Dichter anzunehmen ${ }^{34}$, sodass also der Lykomide das ihm geläufige poetische Element leicht variierend wiederholt verwendet hat. Die dritte Variante (Od. VIII 323) hat noch zwei Parallelen in der Ilias, XV 253 und XXI 461, also an zwei Stellen, die mit Bestimmtheit ebenfalls ihm gehören ${ }^{35}$. Finden sich also alle diese Wendungen ausschliesslich beim Lykomiden, so tritt noch die Hesiodparallele hinzu, die ja nach der Konkordanzenregel wiederum auf die Herkunft aus der musisch-poetischen Orpheustradition weist. Das so in seiner Provenienz festgelegte

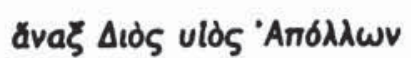

(Od. VIII $334=$ Hes. fr. 235,1)

\footnotetext{
34 Peisistratos 41-60, vgl. bereits Von der Mühll, Hypomnema 303, 33.

${ }^{35}$ Nach Von der Mühll, a.O. 229 f. bezw. 324.
} 
begegnet daher auch noch in den orphischen Schriftpoesien als

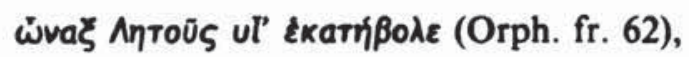

und wiederum zeigt die Orpheustradition noch die ältere Prädikation

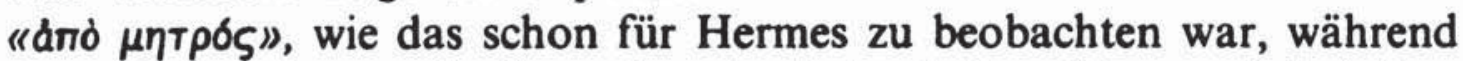
der «Zeus-Sohn» auch diesmal weder beim Lykomiden noch bei dem ganz auf Zeus eingeschworenen Hesiod überraschen kann ${ }^{36}$.

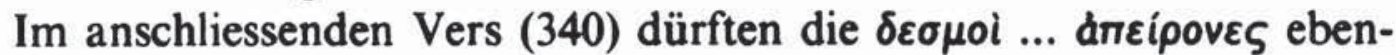
falls eine auf die lykomidische Orpheustradition weisende Prägung sein.

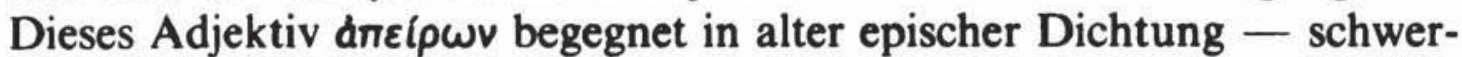
lich zufällig - nur beim Lykomiden und bei Empedokles ${ }^{37}$, wobei die damit gebildeten poetischen Wendungen zugleich ihre Hesiodparallelen haben ${ }^{38}$, sodass es sich nach der Konkordanzenregel um ein der Orpheustradition entstammendes Element handelt, weshalb es ja auch in den orphischen Schriftpoesien seinen angestammten festen Platz hat ${ }^{39}$.

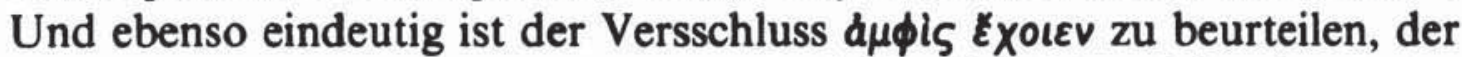
auch in den Formen a $\mu \phi i \varsigma$ हxovtes (Od. III 486, XV 184) oder d $\mu \phi i \varsigma$ Exouoiv (Od. I 54) begegnet, und dies also an Stellen, die wiederum alle dem Lykomiden gehören ${ }^{40}$, wobei das zuletzt genannte in Od. I 54 obendrein sogar in Verbindung mit einem andern bereits bekannten Element der Orpheustradition erscheint:

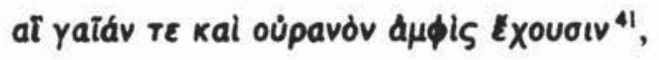

also in einem Kontext, der - neben dem alleinigen Vorkommen der Klausel beim Lykomiden - ebenfalls aufschlussreich sein dürfte: und

${ }^{36} \mathrm{Zu}$ Hesiods Herauslösung des Zeus und der Zeuswelt aus dem alten ("sschamanistischen") Zweieinigen Kosmos s. oben A. 21. - Zum Lykomiden: er dient ja den Bestrebungen des Peisistratos, der als überragendes Zeusheiligtum das (nie vollendete) Olympieion bauen lässt, dazu auch Peisistratos $95 \mathrm{ff}$.

${ }_{37}$ Die Stellen: Il. VII 446 ist eindeutig "B» (Von der Mühll, a.O. 141), XXIV $342,545,776$ sind es ebenfalls, I 350 (in der Lesart fragwürdig - Aristarch war hier vielleicht homerischer als Homer - und in der Zuteilung unsicher), Od. I 98 und V $46=I l$. XXIV 342, Od. XVII 386 und XIX $107=I l$. VII 446, Od. XVII 418, IV 510, Hy. Dem. 173, Aphr. 431. - Emped. VS 31 B 28,1 und 39,1.

${ }_{38}$ Hes. Th. 187, Erga 160, 487, fr. 43,83, 133,1, 204,97. - Th. 678, Sc. 472.

${ }^{39}$ Orph. D.P. 20,3. fr. 1,694. 232,5. 237,7. Hy. 11,20 . 13,4. 59,5. 59,10. 69, 14 .

40 Nach Von der Mühll, hinzuzunehmen freilich Verk. Muse $30 \mathrm{f}$.

${ }^{41}$ Das Taídv re kai Oúpavóv als Element der orpheischen Muse, dem auch Hesiod schon folgt, s. Peisistratos 44. 
der überdies auch noch dadurch gekennzeichnet ist, dass er eine Parallele in der Dichtung des Parmenides hat:

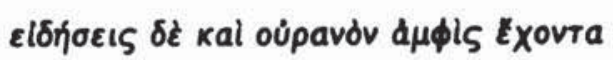

(Fr. 10,5), sodass über den Ursprung auch dieses Elements wirklich kein Zweifel mehr bestehen kann, wenn man weiss, dass bei Parmenides alles was nicht versifizierte Prosa ist - also alles was poetische Parallelen sei es beim "Orpheushypoklepten" Hesiod oder beim Lykomiden oder unmittelbar bei «Orpheus» hat - der präliterarischen Orpheustradition entstammt ${ }^{42}$.

Noch weniger Zweifel kann es für den nächsten Vers geben $(\mathrm{Od}$. VIII 341):

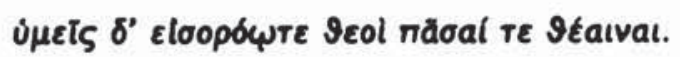

Mit Anfangsvarianten begegnet dies besondere poetische Gebilde mit dem sonst nirgends auftretenden Эéaıvaı ausschliesslich beim Lykomiden:

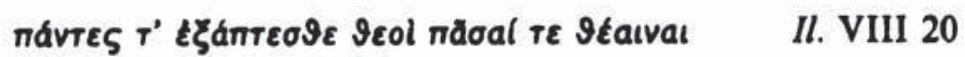

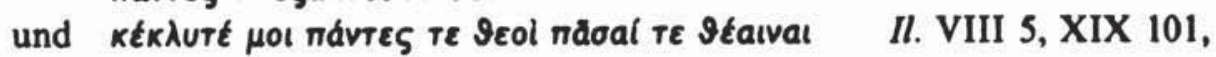

und in dieser in der Ilias sich zweimal findenden Form begegnet es auch noch im Apollonhymnus (v. 311). Auch dieser Vers entstammt der dem Dichter als Gentilerbe vertrauten musischen Orpheustradition, weshalb er sowohl in Il. VIII in unmittelbarer Verbindung mit einer andern Wendung gleicher Provenienz erscheint:

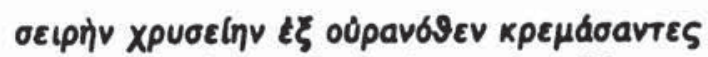

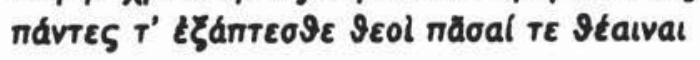

(Il. VIII $19=$ Orph. fr. 166), als auch in "orphischem» Schrifttum seinen Platz hat, zunächst in

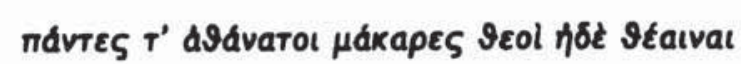

42 Irgendwelche poetischen Parallelen zu «Homer-A» gibt es ja bei Parmenides überhaupt nicht. Hierfür, und überhaupt zur Herkunft seiner Dichtersprache s. Verk. Muse, bes. Teil II und III. 
(Orph. fr. 167b,5), dazu in leichter Variante aber auch in der dem Papyrus-Traktat zu Grunde liegenden Orpheusdichtung:

Toũ $\delta$ ăpa návies

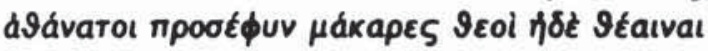

(Col. 12,3f. $)^{43}$. Dabei zeigt auch dieser Vers in seinem jeweiligen Zusammenhang was schon mehrfach zu beobachten war: bei "Orpheus" wie übrigens auch bei Hesiod, Parmenides, Pindar - erscheint noch in mythisch-religiösem Sinn und Kontext, was der Lykomide durchaus profan verwendet ${ }^{44}$.

Die beigezogene Partie 338-341 zeigt also, dass der auf dem Derveni-Papyrus als Orpheusdichtung zitierte Hermes-Vers im DemodokosGesang von Ares und Aphrodite keineswegs allein steht. In dieser Dichtung sind auf engem Raum auch andere Wendungen der gleichen musischen Tradition zum Kontext gefügt. Dieser Sachverhalt ist bereits nicht mehr neu und in den Partien des Lykomiden also auch nicht singulär. Um des Methodischen willen - und auch für das Nachfolgende - ist es an dieser Stelle geboten sich das Wesentliche solcher Kontexte einmal synoptisch zu vergegenwärtigen.

Der analytische Befund, dass es sich im "Homer» in aller Regel um poetische Partien des Letzten Dichters handelt, der - als Lykomide nicht nur Homerisches übernehmen sondern auch aus seiner Gentiltradition dichten kann, geht zumeist auch mit den nicht zu übersehenden Hesiodparallelen zusammen, die den Schluss auf Dichtung aus der musischen Orpheustradition nahe legen. Hinzu tritt dann so gut wie immer

${ }^{43}$ Merkwürdig, dass Kern im Zusammenhang mit Il. VIII 19 nicht auch VIII 20 ( VIII 5, XIX 101, Od. VIII 341) in seinen Index Epicorum ab Orphicis adhibitorum aufgenommen hat, vgl. oben, A. 27. - Die bisher durch Proklos (wohl aus der Rhapsodischen Theogonie) bekannte "Orpheus»-Dichtung (fr. 167) ist jetzt durch den Derveni-Papyrus in einer älteren Form bestätigt, vgl. West, Orphic Poems 267, ist also nicht erstspäten Ursprungs sondern in der Rhapsodischen Theog. aus Altem übernommen: und dürfte sogar, wenn dem lykomidischen Dichter bereits poetische Elemente daraus geläufig sind, zum Ältesten gehören, was aus der präliterarischen Orpheus-Tradition in die Schriftpoesien eingegangen ist.

44 Profanierung der in hereditate sacrorum dem Lykomiden tradierten Muse: Sänger d. Vz. 12 ff., Peisistratos 76-79 und 99-101, emerita 54, 1986, $218 \mathrm{ff}$. - West, Orphic Poems 237 f., will die Abhängigkeit natürlich umgekehrt annehmen: Orph. fr. 166,2 von Il. VIII 19, also die religiös-mythische Aussage von der Unterhaltingsdichtung, sodass der Vers mit dem jungen sekundären Genetiv $\varepsilon \xi$ oúpav $\delta \varepsilon \varepsilon v$ primär wäre. Auf S. 267 setzt er zu den Frr. 164-166 nur «R.» (= Rhaps. Theog.), nicht auch - wie zu 167 - «R.(P.)» (P. = Protogonos Theog.). D.h.: den dem Il. VIII 20 entsprechenden Orpheusvers hält er für alt; die in Verbindung damit auftretende Parallele von Il. VIII 19 dagegen für spät(er)en Ursprungs: "The golden chain is derived from allegorical interpretation of Iliad 8,19" (Vgl. oben, A. 10). 
ein aus dem Kontext nicht zu lösendes Element, das anderwärts ausdrücklich als Orpheus-Dichtung überliefert ist (angesichts des desolaten Zustands unserer "Orphica» ist das erstaunlich oft der Fall): ein Sachverhalt, der die Schlussfolgerung bestätigt. D.h.: «Orpheus»-Verse (bezw. kleinere Elemente) finden sich fast stets in Partien, die durch den

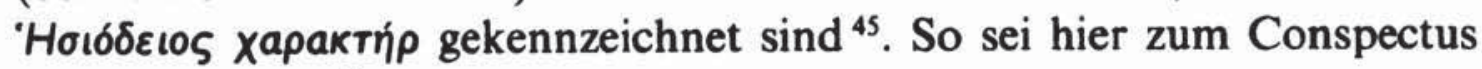
zusammen gestellt:

* In dem in Col. 4,2 zitierten Orpheus-Vers verbindet sich die erste Vershälfte (= Il. V 637) durch seine Variante in Il. XX 305 dem bekannten Orpheusvers XX 308. Der zweite Halbvers, der im "Homer» als Variante erscheint (Il. VIII 236), hat - ebenfalls in Variantenform - hesiodeische Entsprechungen (Th. 534, fr. 141,11, fr. 150,16). Alle betroffenen «Homer»-Stellen finden sich in Partien des Letzten Dichters, und beide Vershälften sind jetzt durch den Papyrus als Elemente der orpheischen Muse ausgewiesen. Bereits dies Beispiel zeigt noch et-

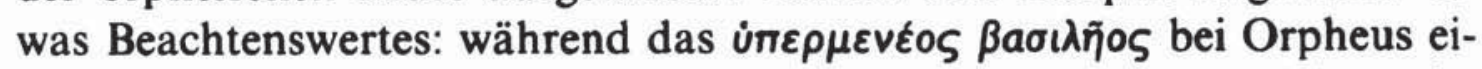
nem religiösen Kontext angehört und den höchsten Gott bezeichnet, ist diese poetische Wendung vom Lykomiden profan gebraucht.

* In der poetischen Herakles-Assoziation (Hy. XV und Nekyia 602 ff.) handelt es sich wiederum um Dichtung des Lykomiden, und gleichzeitig gibt es die nicht zu übersehenden Hesiodparallelen, die den Schluss auf poetische Elemente der Orpheustradition nahe legen. Wollte man der antiken Kritik, die den Nekyia-Passus dem Onomakritos zuschrieb, keine grosse Bedeutung beimessen, so kommt jetzt der Derveni-Papyrus hinzu, auf dem der aus der Assoziation nicht zu lösende Vers Hy. XV 7 als Orpheusvers zitiert ist.

* Der ausführlicher besprochene Hermes-Vers (Od. VIII 335) findet sich im «Homer» in einer dem Lykomiden gehörigen Partie. Hesiodparallelen zu beiden Halbversen (und ihren Varianten) lassen auf Orpheus schliessen, wie ja beide Halbverse als poetisches Gut der Orpheustradition sich bis in die orphischen Hymnen erhalten haben. Wem die späten orphischen Hymnen kein ausreichender Beweis sind ${ }^{46}$, der hat diesen jetzt auf dem Derveni-Papyrus. Und dass auch dieser Vers vom Lykomiden profaniert ist (dass er in einer schwankhaften Erzählung «komische Wirkung» erzielt), bedarf keiner besonderen Hervorhebung mehr.

* Im Zusammenhang mit dem Hermes-Vers findet sich in Od. VIII auch die Fügung «alle Götter und Göttinnen», eine durch das besonde-

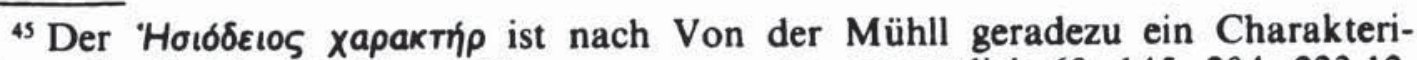
stikum des Letzten Dichters: Hypomnema passim, namentlich 60, 145, 204, 223,12, $224,385$.

46 Dazu jedoch oben, A. 14. 
re Wort Séaıva unverwechselbare Prägung. Sie findet sich im "Homer» insgesamt fünf Mal, auch sie ausschliesslich in Partien des Letzten Dichters (neben Od. VIII 341 noch Il. VIII 5, 20, XIX 101, Hy. Ap. 311). In Od. VIII steht sie wiederum nicht allein sondern gehört einem Kontext an, in dem ja auch anderes der Orpheustradition entstammt (oben S. 223 ff.). Doch lässt sich dieser Fall noch sicherer beurteilen, wenn man gleich das Folgende hinzunimmt:

* Im Anfang des $I l$. VIII - in Dichtung die ganz dem Letzten Dichter, dem Lykomiden, gehört - findet sich in der Partie 12-20 eine ganze Reihe von Wendungen der Orpheustradition zum Kontext gefügt. $\mathrm{Da}$ das an anderer Stelle bereits gezeigt ist ${ }^{47}$, bleibt hier nur festzuhalten, dass es auch hier mehrere Parallelen beim "Orpheus-Hypoklepten» Hesiod sind, die den Schluss auf die altorpheische Muse erlauben (während der Annahme einer direkten Hesiodabhängigkeit doch manches entgegensteht) ${ }^{48}$. Die Bestätigung stellt sich sofort ein: (a) Eine dieser Hesiodkonkordanzen (Il. VIII $13 \mathrm{~b}=$ Hes. Th. $736 \mathrm{~b}, 807 \mathrm{~b}$, u.a.) ist durch Orph. Hy. 56,10 auch als Element der Orpheustradition bezeugt. (b) Der im Il. VIII-Eingang zweimal applizierte Vers von «allen Göttern und Göttinnen" bildet an der Stelle seines zweiten Vorkommens mit dem vorangehenden eine Einheit, wobei dieser vorangehende seinerseits in den orphischen Literaturpoesien eine Entsprechung hat (Orph. fr. 166,2). (c) Schliesslich ist der mit dem Téaıvaı geprägte Vers selbst ausreichend als Orpheusvers bezeugt: Orph. fr. 167b,5 und dazu jetzt auf dem Papyrus, Col. 12,3 f.

* Im Anfang der Nekyia zeigt die Partie 13-19 den gleichen Befund wie der Anfang des Il. VIII. Auch da gibt es durchgehend Hesiodparallelen, zu dessen Unterweltspartie natürlich, ohne dass an Hesiodbenutzung zu denken wäre: man müsste ja z.B. annehmen, dass der Dichter sich zwar eng an Hesiods Vorbilddichtung in der entsprechenden Theogoniepartie gehalten hätte, dass er dazwischen aber auch einen vom Böoter eigens zur Bezeichnung der Neger geschaffenen Vers aus den $\operatorname{Erga}$ (v. 527) beigezogen und ihn auf Unterweltliches (die «Kimmerier») umgedeutet hätte, um ihn unter die Theogoniereminiszenzen einfügen zu können ${ }^{49}$.

${ }^{47}$ Peisistratos $32 \mathrm{ff}$. , bes. 35-37.

48 Früher hat man - als vielbeachteter Fortschritt gegenüber dem anfänglichen Glauben an die uneingeschränkte Priorität Homers - die Il. VIII-Partie also von Hesiod abhängig erklärt: so z.B. Bethe, Hom. II 2, $307 \mathrm{ff}$ (bes. $311 \mathrm{ff}$.), Friedrich Schwenn, Die Theogonie des Hesiodos, Heidelberg 1934, 66 ff. - Zur neuen Erklärung s. Peisistratos a.O. (vorige Anm.).

${ }_{49} \mathrm{Zu} \mathrm{Od}$. XI 13-19 jetzt Peisistratos 32-35. Zum «Negervers» als der mythischpoetischen Unterweltsassoziation entstammend Orpheus 42-45, anerkannt von Von 
* In der Dichtung vom Phäakenpalast (Od. VII 78-132) gewahrt man abermals das Gleiche: «Orpheus»-Elemente zusammen mit Hesiodreminiszenzen, die indessen auch hier keine Hesiodbenutzung sein können. Dass auch diese Dichtung eindeutig dem Letzten Dichter gehört, ist selbstverständlich. Und dass er aus seinem Gentilerbe schöpfend gedichtet hat, ergibt hier einen besonders eindrucksvollen Befund. Dem Seher $=$ und Priestergeschlecht der Lykomiden war ja namentlich alttradiertes musisches Gut aus der Demeter-Assoziation zu eigen (auf Grund dessen dieser Dichter ja auch den "homerischen» Demeterhymnus geschaffen hat) ${ }^{\text {so }}$. In der Partie vom Phäakenpalast greift er just Elemente dieser Demeter-Assoziation auf, deren Sekundärverwendung hier nur allzu offensichtlich ist. Erinnert sei hier an die "eherne Schwelle» sonst mythischer Übergang zur Jenseitswelt - , die hier ganz profan als Schwelle des Palastportals erscheint (Od. VII 83 und 89), Tod = und Alterslosigkeit, die zu verleihen zu den Segnungen der Göttin gehört, kommt hier den aus Edelmetall gefertigten Hunden zu, die zu beiden Seiten des Portals angebracht sind (VII 94), brennende Fackeln in den Händen irrt die nach ihrer geraubten Tochter suchende Göttin umher (eine Kult-Ätiologie), während im Alkinoospalast goldene Jünglinge auf Sockeln aufgestellt sind, mit brennenden Fackeln in den Händen: als aparte Saalbeleuchtung beim Bankett (VII 100 f.). Zu solchem "homerischen" Fabulieren hat er das musische Gentilerbe übel profaniert ${ }^{51}$. (Der Hermes-Vers im Demodokos-Gesang steht also wirklich nicht allein).

* Auch anderwärts lässt sich das Gleiche beobachten: Elemente in Partien des Letzten Dichters, meist mit Hesiodreminiszenzen, die keine Hesiodbenutzung sein können (z.B. Od. X 86 gegenüber Hes. Th. 748), die aber der mythischen Demeter-Assoziation (also dem lykomidischen Gentilerbe) entstammen und in den Grossepen profaniert erscheinen, wie z.B. Il. VIII 46, V 499-501, XV 121 f. ${ }^{52}$ Der letzte dieser Fälle zeigt, dass Thema und Leitmotiv des Demetermythus, nämlich der Göttin Groll den Unsterblichen gegenüber ob des Verschwindens ihrer Toch-

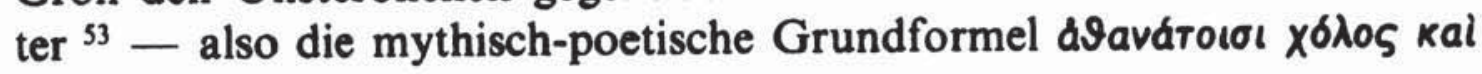

der Mühll, Kl. Schr. 160,13 - Wie Hesiod mit der ihm überkommenen Muse umge-

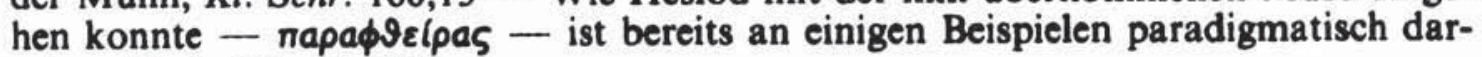
getan: Verk. Muse $142 \mathrm{f}$.

so Peisistratos 16-27 (bes. 20 f.), dazu 74-77. (Dort müsste es auf S. 22 Zeile $8 \mathrm{f}$. freilich heissen, dass die Hekate selasphoros «in ganz Griechenland unter dieser Bezeichnung nirgends begegnet ausser...").

${ }_{51}$ Für diese Profanierung s. emerita 54, 1986, 218 ff., bes. 221-226.

32 Diese Stellen sind in "Alpha 1" behandelt, emerita 227-232.

53 Als Kern-Element des Demetermythos durchzieht das Groll-Motiv das Ganze: Hy. Dem. 82 f. 91,303 ff., 329 f., 338 f., 349 ff., 354 f., 409 f., 451 ff. 
$\mu \tilde{\eta} v ı \varsigma$ - im Grossepos sekundär verwendet ist zu dem irrealen Gedankenspiel, dass Zorn und Groll des von seiner Gemahlin überlisteten Zeus den Unsterblichen gegenüber noch schlimmer geworden wäre, hätte nicht Athene eingegriffen: dass also Il. XV 122 durch Verwendung der einem Lykomiden stets gegenwärtigen Grundformel des Demeterhymnus ( $350 \mathrm{f}$. und 410 ) geschaffen ist ${ }^{54}$, woraus sich dann auch von selbst ergibt, dass ebenso auch Il. I 1 nach eben dieser Menis der Göttin gebildet, also auf den Groll des Sterblichen übertragen und somit wiederum profaniert ist ${ }^{s 5}$.

* $\mathrm{Zu}$ den Hesiodkonkordanzen ist schliesslich noch ein paradigmatischer Sachverhalt anzuschliessen. Notiert man im «homerischen» Demeterhymnus sämtliche feststellbaren Hesiodkonkordanzen, so betrifft das etwa ein halbes Hundert Verse bezw. Versteile dieses Hymnus, die - nach der inzwischen nicht mehr ganz unbegründeten Theorie - der alten Orpheustradition zuzuschreiben sind ${ }^{56}$. Und die so ermittelten poetischen Elemente bilden stellenweise ganze geschlossene Kontexte. Der unerforschliche Zufall will es nun, dass von fünf solchen Passagen nicht weniger als deren vier auf dem Berliner Papyrus als Orpheusdichtung zitiert werden. Eine bessere Bestätigung der Richtigkeit unserer Auffassung von den "homerisch»-hesiodeischen Konkordanzen kann man sich nicht wünschen. (Dabei ist der «homerische» Demeterhymnus ja diejenige Schöpfung, in der sich ihr Dichter unfreiwillig als Lykomide verrät ${ }^{57}$, ohne dass sich hier - beim "Thema" des Hymnus begreiflicherweise - seine sonst zu beobachtende Profanation der altgeheiligten Muse bemerkbar machte).

${ }^{54}$ Das Umgekehrte anzunehmen - dass Kernvorstellung und mythisch-poetisches Leitmotiv des Demeterhymnus Derivat aus diesem irrealen Gedanken in der Erzählung von dem ob der List seiner Gemahlin aufgebrachten Zeus sei - : das anzunehmen überlassen wir den modernen Mythologen, die heute wieder verkünden, dass Homer - Ilias und Odyssee - um die Wende vom achten zum siebenten Jahrhundert schriftlich fertig vorgelegen habe, und dass alle andern - alle kyklische Dichtung (also auch die Aithiopis), Hesiod, die Hymnen, die Lyrik, Xenophanes und Parmenides, selbstverständlich auch "Orpheus", usw., aus diesem Homer das Ihrige bezogen haben. Aus Il. XV 122 hat sich also das Urmotiv des Demetermythos und

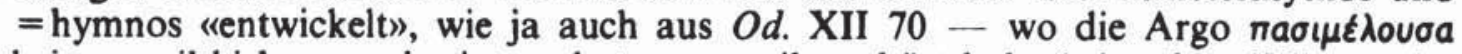
heisse, weil bislang noch niemand etwas von ihr gehört habe (wie schon Wilamowitz spottete) - sich der Argonautenmythos «entwickelt» habe...

55 Die entsprechende antike Uberlieferung kann also nicht verworfen werden, vgl. "Alpha 1", EMERITA $233 \mathrm{ff}$. (Einschlägiges zum Menis-Vers bereits Orpheus $58 \mathrm{ff}$., bes. 61-66.)

${ }_{36}$ Das ist schon dargelegt, Orpheus 75-111 (beachte bes. 111,7 den Hinweis auf die alte Arbeit von Kuno Francke), dazu Peisistratos $19 \mathrm{f}$.

${ }_{57}$ Zum Lykomiden zunächst oben, A. 26. Hinzuzunehmen die Bewährung dieser Identifikation in der Erklärung des Bestandes an poetischen Parallelen zur Parmenideischen Dichtersprache: Die Verkannte Muse 85 mit 94-98. 
Die Gleichläufigkeit in all den genannten Dichtungspartien ist allzu konsistent und eindeutig, als dass sie entweder übersehen oder als Häufung von «Zufällen» erklärt werden könnte. Die Lösung eines alten Problems - der Interrelation zwischen Hesiod, «Homer" und "Orpheus» - zeichnet sich $\mathrm{ab}^{58}$.

Wenden wir uns nun aber zu den beiden verbleibenden Orpheusversen des Derveni-Papyrus, die bisher - aus Il. XXIV $527 \mathrm{f}$. - nur als «Homer»-Verse bekannt waren. In deren homerischem Kontext trifft man sogleich wieder auf die bekannten Sachverhalte. Die ganze Il. XXIV-Dichtung gehört ja zum Spätesten im «Homer», ist nach der Analyse eindeutig das Werk des Letzten Dichters, des Lykomiden, der ja auch das $\boldsymbol{O d}$. VIII geschaffen hat ${ }^{59}$. Wiederum dürfte der Sachverhalt der sein, dass er aus seiner Gentiltradition auch hier mythisch-poetisches Gut der orpheischen Muse hat einfliessen lassen. In dieser Auffassung bestärkt dann der wiederum hesiodoide Charakter der Dichtung. In unmittelbarer Umgebung (v. 525) findet sich zunächst das

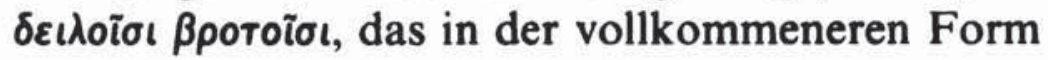

$$
\pi \varepsilon \dot{\lambda \varepsilon t a \iota ~ \delta \varepsilon \iota \lambda о і ̈ \sigma \iota ~ \beta р о т о і ̈ \sigma ı(v) ~}
$$

auch Hesiod schon kennt (Erga 686), und das der Letzte Dichter leicht variiert mehrfach verwendet

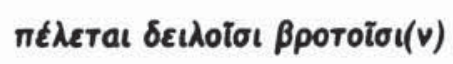

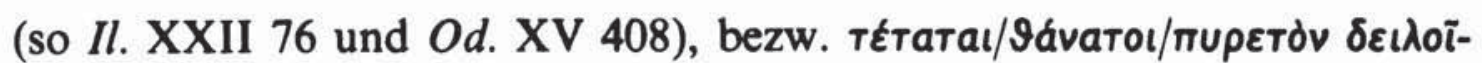

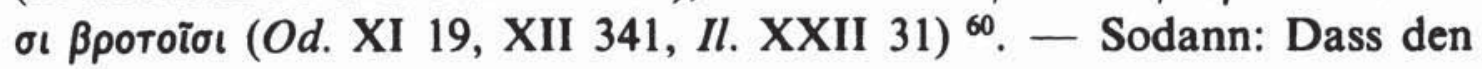

s8 Mit der gegebenen rekapitulierenden Zusammenordnung der bereits behandelten Partien ist sicherlich noch lange nicht alles erfasst. Eine systematische Untersuchung aller «B»-Partien wird unverzichtbar werden. Doch sind wir von einer wissenschaftlichen Diskussion des sich klar genug abzeichnenden Problems im Augenblick noch weit entfernt.

39 «Da der Dichter (sc. des Il. XXIV) aber zum Teil auch aus den anerkannt jüngsten Teilen der Ilias schöpft und mehrfach auch mit der Odyssee sich nahe berührt, ist ein verhältnismässig junger Ursprung des Gedichts mit Sicherheit zu erschliessen... Hiernach können wir nur mit den meisten Neueren annehmen, dass der 24. Gesang ursprünglich der Ilias fremd war und wie der 23. seinen Ursprung vielmehr einem jüngeren Dichter verdankt...", Ameis-Hentze, Anhang, 8 . Heft, $94 \mathrm{f}$. Vgl. dazu auch oben, A. 17. "That the book as a whole is late admits of no doubt...", Leaf, Introd. to Il. XXIV (p. 536). Es gehört also "B» nach Von der Mühll. - Zur Identität des "B»" in Il. und Od. s. Von der Mühll, Hypomnema 59, 141 ff., 204, 351, 374, ebenso H. Mühlestein, SMEA 25, 1984, 323-27. Vgl. Orpheus 57-59, Peisistratos 41-60.

${ }^{60}$ Das $\pi \varepsilon \lambda \varepsilon t a \iota$ findet sich in Il. XXIV im gerade vorangehenden Vers 524 in gleicher Position: zu diesem Auseinandernehmen des Ausdrucks vgl. das ... alvótatov ... kal kúvtatov in Hy. Dem. $305 \mathrm{f}$. (Orpheus 98). - Zu den genannten Stellen s. Von der Mühll, auch Il. XXII 29-31 (oder 30-31) ist deutlich als Einschub kenntlich (obwohl bei Von der Mühll zufällig nicht ausdrücklich vermerkt). 


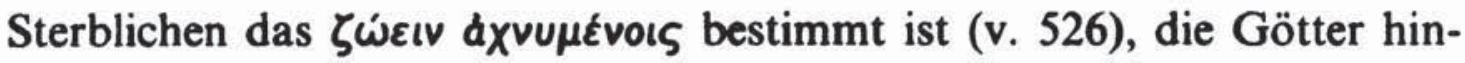

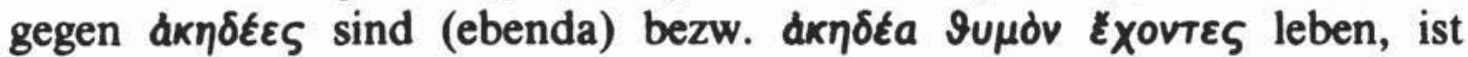
ebenfalls hesiodeisch, der von den Menschen des Goldenen Zeitalters gerade dies aussagt, dass sie

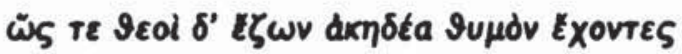

(Erga 112), wie auch den göttlichen Kindern des Zeus und der Mne-

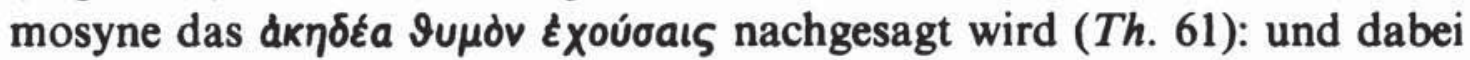
gibt die zweimalige Verwendung dieses poetischen Elements in verschiedenen Zusammenhängen die dem Hesiod bereits tradierte Muse zu erkennen. Und deren mit "Homer» konkordante poetischen Elemente sind auch hier Orpheusprägungen. Sieht man von weiteren hesiodoiden Elementen in der weiteren Umgebung vollkommen ${ }^{a b}{ }^{61}$, so tritt dem Verspaar von den zwei Pithoi ebenfalls Hesiodeisches zur Seite: «Die Verbindung eines hesiodeischen Motivs mit hesiodeischer Wortwahl begegnet auch einmal im (... späten) XXIV der Ilias. Il. XXIV 527 ist die Rede von den beiden Fässern des Zeus, aus denen der Gott Gutes und Böses verteilt. 'Eáwv findet sich hier, und zwar innerhalb der Ilias nur hier, die Form ist der Genet. Plur. Femin. wie bei Hesiodos Th. 633, 664. Das Motiv der ni $(90$ - auch dies Wort begegnet in der Ilias nur hier - erinnert ohne Weiteres an den nío५ der Pandora aus den Erga (v. $94 \mathrm{ff}$.)" ${ }^{62}$. Es «erinnert» fraglos an Hesiod: wollte man jedoch annehmen, der Letzte Dichter habe in seinem Il. XXIV-Passus Hesiod benutzt ${ }^{63}$, so tritt das Unwahrscheinliche sofort zu Tage. Pandora, dies

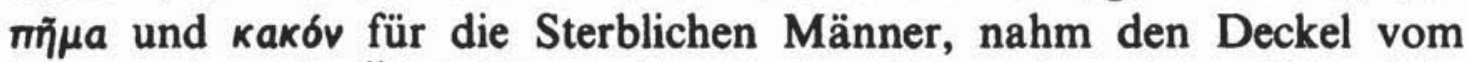

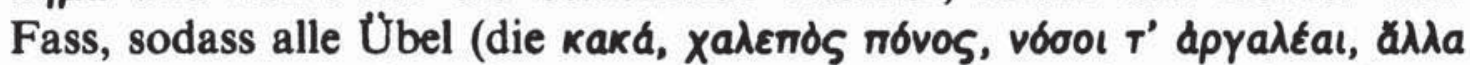

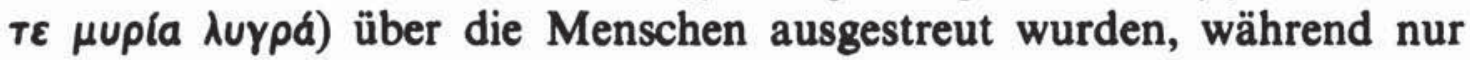
die Hoffnung drinnen blieb. Der Letzte Dichter hätte dann in seinem Il. XXIV aus diesem Fass der Pandora die zwei Fässer des Zeus selbst erfunden... und nicht nur das: das besondere $\varepsilon a ́ \omega v$, das der Pandora-Erwähnung und den Erga überhaupt fremd ist, hätte er dem in der Theo-

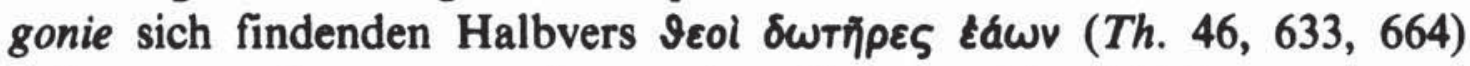
entnommen, da er es aus einer andern Homerpartie nicht kennen konnte (die beiden Vorkommen an der besprochenen Od. VIII-Stelle gehören

${ }^{61}$ So etwa kúperal, $530 \sim$ Hes. Erga 691 , Evtòs képyel, $544=$ Hes. Th. 751,

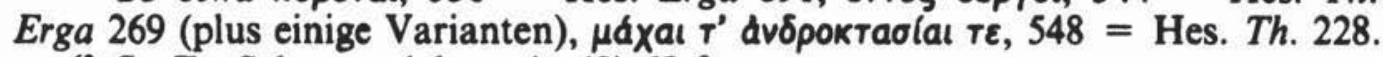

${ }_{62}$ So Fr. Schwenn (oben, A. 48) $68 \mathrm{f}$.

${ }^{63}$ Schwenn musste das annehmen, da eine Umkehrung des Verhältnisses allerdings unmöglich ist und die Frage nach gemeinsamem Ursprung garnicht gestellt werden konnte, wenn es als selbstverständlich galt, dass es ausser Homer und Hesiod noch keine andere Epik gegeben haben könne. 
ja ihm selbst). Das Unwahrscheinliche solchen Zusammenstückens entlegener Elemente ist bereits am «Negervers» innerhalb der Jenseitsassoziation deutlich geworden, ist aber auch anderwärts bereits gesehen ${ }^{64}$.

Also: hesiodoide Dichtung ohne jedoch Hesiodentlehnung zu sein, und das in einer Partie, die dem Letzten Dichter, dem Lykomiden, gehört, dem Angehörigen eines Geschlechts also, über dessen alte Orpheustradition ohnehin kein Zweifel möglich ist ${ }^{65}$, dazu die mehrfach erprobte fundamentale Schlussfolgerung, wonach in "homerisch"-hesiodeischen Konkordanzen Reste alter Orpheus-Tradition vorliegen ${ }^{66}$ : das lässt den sicheren Schluss zu, dass Il. XXIV 527 f. der musisch-poetischen Orpheustradition entstammen ${ }^{67}$.

Finden sich die Verse nun tatsächlich in Orpheusdichtung, nämlich in jener bereits literarisch fixierten «Orpheus»-Dichtung, über die der Autor des Papyrustraktats verfügt, so beweist das nur, dass die angewandte Methodos mitsamt ihrer Schlussfolgerung richtig ist.

Ist dem so, so lernen wir abermals etwas über den eigenwilligen Böoter. Hesiod hat ja nicht nur únoк

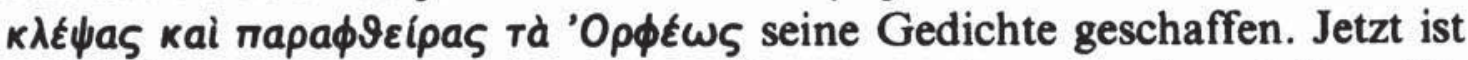
ersichtlich, dass er einer ihn beherrschenden misogynen Assoziation folgend - nämlich: "Weib $=$ kakóv $=$ kaká $=\pi(\vartheta \circ \varsigma$ kakw̃v» in Verbindung mit einem komplementären $" W e i b=$ Neugier $=\pi \tilde{\omega} \mu^{\prime}$ ' $\phi \phi \varepsilon \lambda \circ \tilde{\sigma a}$ » -, dass er also eigener Einsicht folgend von den beiden Fässern des Zeus nur das der Úbel aufgegriffen, aus den zwei Fässern des Zeus somit nur das Eine der Pandora gemacht hat. Das ergab eine gewisse Unlogik, weil ja aus dem Fass, von der Hoffnung abgesehen, alle Úbel ausgestreut werden, ohne dass noch gesagt wäre, woher denn die guten

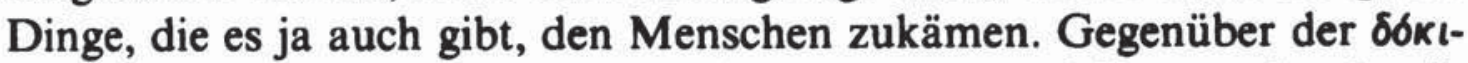

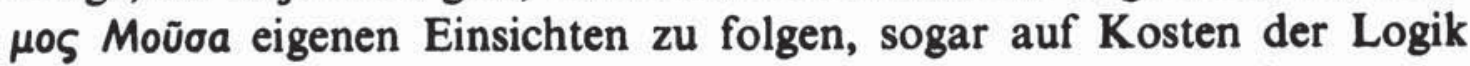
oder Ausgewogenheit: dieser Sachverhalt dürfte dem zuzurechnen sein,

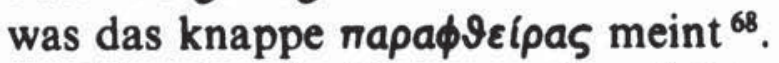

Wer also der Homeranalyse folgt und im Letzten Dichter den Lyko-

${ }^{64}$ So z.B. von Hoekstra, Mnemosyne 10, 1957, $139 \mathrm{ff}$.

65 Peisistratos 69 ff., bes. 74-77.

66 s. oben, A. 15.

${ }^{67}$ Hesiods beigezogener Halbvers, Th. 46, 633, 664, erweist sich durch die mehrfache Verwendung als ein ihm bereits überkommenes Element. - Auch ein anderer im Traktat zitierter Orpheusvers hat seine signifikanten Parallelen: Col. 4,4 $\sim O d$. XI $151(=$ «B» = L) und Hes. fr. 193,8 (wo wahrscheinlich 9eoфar' dxoúoas zu lesen ist, s. PSI vol. 2, 1913, No. 131, ed. Medea Norsa, die zu fr. 193,12 auch bemerkt: "Non ci maraviglieremmo se ci fosse l'intero verso omerico [II. VIII 291]....").

${ }_{68} \mathrm{Zu}$ Hesiods Verhältnis zur überkommenen Muse s. oben A. 49, hinzuzunehmen der fragwürdig gewordene Anfang seiner Theogonie, Verk. Muse 141. 
miden am Werk weiss, wird in der ganz ihm gehörenden Dichtung ohnehin mit Elementen der musischen Orpheustradition rechnen, und wenn er bereit ist die inzwischen ausreichend erprobte fundamentale Schlussfolgerung zu den "homerisch»-hesiodeischen Konkordanzen anzuerkennen, ergibt sich ihm zwangsläufig, dass wie in so manchem zuvor Besprochenen auch in dem Mythologem von den beiden Pithoi alttradierte Orpheusdichtung vorliegen müsse. Da die Homeranalyse und die Identifikation des Letzten Dichters ebenso wie auch der «Fundamentalschluss" schon vor dem Bekanntwerden des Derveni-Papyrus vorgelegen haben, sollte man nun eher dieses dreiteilige Instrumentarium als gerechtfertigt und bewährt anerkennen, anstatt weiterhin die der gesamten Antike zuwiderlaufende - und unbewiesene wie auch unbeweisbare - These von den sogenannten "Orphikerplagiaten" aus dem literarischen Epos aufrecht erhalten ${ }^{69}$.

Gegenüber einem der drei Teile des Instrumentariums könnte zwar ein Einwand erhoben werden. Denn der «Fundamentalschluss», wonach in «homerisch»-hesiodeischen Konkordanzen Elemente der alten präliterarischen Orpheustradition zu fassen sind, ist ja doch zunächst nur ein auf überaus schmaler Basis errichteter Induktivschluss, also so fragwürdig und unzuverlässig wie derartige Schlüsse nun einmal sind ${ }^{70}$. Doch dieser Einwand hat sich inzwischen erledigt. Wenn nämlich - einerseits - der Letzte Dichter am "Homer» jetzt als Lykomide erkannt ist, also als Angehöriger eines Geschlechts dessen alte Orpheustradition sich keinesfalls in Frage stellen lässt, und wenn - andererseits

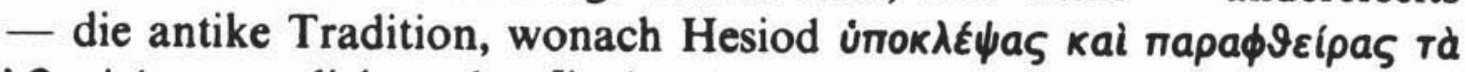
' $0 \rho \phi \varepsilon \omega \varsigma$ gedichtet hat ${ }^{71}$, in dem Augenblick anerkannt werden

${ }^{69}$ Wer an die "plagiierenden Orphiker» glaubt - und es könnten aus der neuesten Literatur genug Namen genannt werden - wird einige ihm wenig angenehme Dinge anerkennen müssen: $\mathbf{z . B}$. diese «Fälscher» haben sich - mirabile dictu - an die Homeranalyse gehalten, um nur die «B»-Partien zu plündern. Sie haben dabei in Od. X 427 anstatt der beiden jüngeren Komparative auf auf -i $\omega v$ eingesetzt, um Alter vorzutäuschen, haben - wiederum um Alter vorzutäuschen - in den dem Demeterhymnus entnommenen Passagen Varianten mit noch wirksamem Digamma geschaffen, haben sonst noch sehr Altertümliches aufgestöbert, z.B. dpyúфeos (fr. 70), haben den dem $\mathrm{Il}$. V entnommenen Passus (fr. 268) überhaupt verbessert (s. Peisistratos $50 \mathrm{f}$.): sie müssen also zum Fälschen versierte Philologen (die es noch nicht gab) herangezogen haben. Dieses Streben nach Altertümlichkeit widerstreitet zwar ihrem Vorsatz, nur junge Homerpartien zu plündern - aber darf man "gerissenen Fälschern» Inkonsequenz vorwerfen?

${ }^{70}$ Das Problematische des Induktiv-Schlusses ist gleich bei seiner ersten Formulierung nicht verschwiegen: Orpheus 38,2. Vgl. weiter auch Peisistratos $30 \mathrm{ff}$. mit 32,4 .

7 Schol. Lycophr. 399 p. 149,13 Scheer ( = Kern, O.F. test. 246), dazu EMERITA 19, 1951, 107 ff., Orpheus 49,5, Sänger d. Vz. $16 \mathrm{f}$. 
muss, wo auch nur Ein überzeugender Fall von Vorhergang orpheischer Muse vor Hesiod aufweisbar ist (und diesen hat bereits Mazon aufgezeigt, indem er die theogonischen Anfänge - besonders den Eros - bei Hesiod und Orpheus verglich ${ }^{72}$, von zusätzlichen weiteren gleichartigen Fällen kann zunächst ganz abgesehen werden) - : aus diesen beiden Gegebenheiten resultiert von selbst, dass die «homerisch»-hesiodeischen Konkordanzen - die sich ja nur in Lykomidenpartien finden - Relikte der den beiden Dichtern, dem Lykomiden wie dem Böoter, gleichermassen vertrauten älteren Orpheustradition sind, ergibt sich also, dass der «Fundamentalschluss» nun auch deduktiv gerechtfertigt ist (sodass man die schmale Basis des ursprünglichen Induktivschlusses jetzt vergessen kann).

Was also zunächst vielfach - am deutlichsten etwa am Demeterhymnus - empirisch-experimentell festzustellen war (s. oben), erhält jetzt seine definitive Begründung aus der Tradition, die den beiden Dichtern vertraut ist, der präliterarischen Orpheustradition, die bei keinem von beiden in Zweifel gezogen werden kann ${ }^{73}$.

Diese jetzige Erklärung findet gleich auch noch doppelte Bestätigung. Die erste liegt darin, dass die vermeintlichen "Orphikerplagiate", also die Homer und Orpheus gemeinsamen Verse, sich stets in solchen

${ }^{72}$ Hésiode, éd. P. Mazon, Coll. Budé, 1928, p. 27, Sänger d. Vz. 17-20. Vgl. auch Verk. Muse $142 \mathrm{f}$.

${ }_{73}$ Ist der Letzte Dichter am Homer als Lykomide identifiziert (wie im Peisistratos dargelegt), so führt das nicht nur zur jetzigen Erklärung der hesiodeisch-homerischen Konkordanzen und damit zum Verständnis der poetischen Orpheus-Elemente im "Homer», angefangen von Alpha 1, es wird aus der Herkunft und Tradition des Lykomidengeschlechts auch anderes unmittelbar aufgeklärt: $\mathbf{z . B}$. des Letzten Dichters unverkennbare Vorliebe für die Neleiden und das Neleische Pylos, besonders Nestor (SMEA 25, 1984, 332, 39), dazu die andern messenischen Traditionen, denen er folgt oder die er gar bei der Gestaltung der Grossepen eigens einbezieht (so etwa Machaon und die Machaoniden, dazu der in Ilias und Odyssee und Demeterhymnus nicht weniger als sechsmal eingeflochtene Diokles), und vollends unübersehbar tritt seine Vorliebe für Messenisches dann hervor, wenn eine solche Tradition sonst nicht anerkannt ist, weil sie im Gegensatz zur prominenteren und vom delphischen Gott sanktionierten Version steht (so die Abstammung des Asklepios). Die Bindung der Lykomiden an Messenien hat sowohl im Mythos des 6. Jhdts. als auch später historisch ihren Ausdruck gefunden, letzteres in der Entsendung des Methapos zur Reorganisation der Mysterien von Andania. ( $\mathrm{Zu}$ diesen Zusammenhängen: Orpheus 314 f., 317. Peisistratos 72 f., 80-84. H. Mühlestein, SMEA 9, 1969, 70 f.; 17, 1976, 137 ff.; 25, 1984, 323 ff.). - Dass sich mit der Identifizierung des Letzten Dichters spontan Simultanlösungen für mehrere so verschieden geartete Probleme einstellen: das verdient hier am Rande vermerkt zu werden, um bei einem mit den einschlägigen Zusammenhängen nicht vertrauten Leser nicht den Eindruck aufkommen zu lassen, als ob diese Identifizierung eine ad-hoc-Konstruktion d.h. ein deus ex machina sei, erfunden lediglich um die Lobeck-Kernsche Theorie aus den Angeln heben zu können. 
Partien finden, in denen es gleichzeitig auch die Hesiodreminiszenzen und $=$ konkordanzen gibt. (Warum sollten "plagiierende Orphiker" sich gerade an solche Partien gehalten haben, die durch den Hoı́ócıs Xaракти́p gekennzeichnet sind?). Die zweite liegt darin, dass die aus den homerisch-hesiodeischen Konkordanzen erschlossenen Elemente überaus häufig dadurch ausgewiesen sind, dass sie sich bis in die aus der mündlichen Tradition hervorgegangenen Schriftpoesien erhalten haben, uns also unter dem Namen "Orpheus» auch bezeugt sind. Und auch diese Bezeugung ist ihrerseits wiederum genügend ausgewiesen. Wenn z.B. aus den Konkordanzen einige Kontexte aus der Demeter-Assoziation als "Orpheus»-Dichtung zunächst erschlossen sind, das Erschlossene auf dem Berliner Papyrus (Orph. fr. 49) als Orpheusdichtung auch bestätigt ist (oben S. 230), so tritt der entscheidende Befund hinzu, dass diese Zitationen eine altertümlichere Form aufweisen als die betreffenden Stellen des «homerischen» Demeterhymnus, womit jede (aus der Lobeck-Kernschen Theorie inspirierte) Spekulation, ob die Orpheuszitate nicht vielleicht doch dem "Homer» entnommen sein könnten, hinfällig wird ${ }^{74}$. Steht aber als Kriterium die altertümlichere Form nicht zur Verfügung (da sich ja die "orphischen» Schriftpoesien auch weiterentwickelt haben), so gibt es noch eine andere Möglichkeit die relative Anciennität festzustellen: die gleichlautenden Elemente sind im "Homer» durch Profanierung und Trivialisierung deutlich in ihrer Sekundärverwendung zu erkennen: Paradigmata hierfür enthält ja die Dichtung vom Phäakenpalast ${ }^{75}$. (Dass auch diese Art der Bestätigung nicht in jedem einzelnen Fall vorliegen kann, dürfte beim desolaten Erhaltungzustand der Orphica einleuchten.)

Die jetzt gewonnene Erklärung der «homerisch»-hesiodeischen Konkordanzen ist weit überzeugender als alle bisherigen Versuche Hesiod von Homer (von Il. VIII und XXIV, von Od. X-XI und VIII, usw.) oder auch umgekehrt Homer von Hesiod herleiten zu wollen. Ein ganzes Jahrhundert philologischer Bemühung hat ja eine unmittelbare Abhängigkeit des Einen vom Andern nicht überzeugend nachweisen kön-

74 Dargelegt bereits Orpheus $103 \mathrm{ff}$. mit der (allein schon aufschlussreichen) Ergänzung Peisistratos $20 \mathrm{f}$. - Das Gleiche zeigt auch Orph. fr. 234 gegenüber $O d$. XI 427 (vgl. Hes. Erga 702 f.), wo "Orpheus» noch den alten Komparativ plyıov hat, «Homer» dagegen nur die beiden jüngeren Komparative auf -répos, Peisistratos 32.

75 S. emerir 54, 1986, 203 ff., bes. 215 ff. Ebenso ist z.B. Parm. 1,11 - der Orpheustradition folgend - morphologisch älter als das triviale Od. X 86, Peisistratos 31. Hierher gehören auch Il. V $637+$ VIII 236, V 599 ff., Od. VIII 335, Il. VIII 45 f., XV 122 mitsamt I 1 (oben, S. 229 f.). 
nen ${ }^{76}$. Der alte und zähe Glaube an eine solche wird inzwischen von namhaften Forschern nicht mehr geteilt ${ }^{77}$. Für den hinter Hesiod und "Homer» zurückliegenden gemeinsamen Ursprung ist vielmehr bereits die mykenische Zeit anvisiert und eine gemeinsame mykenische $\mathrm{Q}$ uelle postuliert ${ }^{78}$. Und dem entspricht uneingeschränkt unsere jetzige Erklärung, wonach es Relikte der alten musischen Orpheustradition sind, die in den "homerisch»-hesiodeischen Konkordanzen vorliegen ${ }^{79}$. Denn die anfänglichen Träger dieser musischen Tradition fasst man in Neleus und den Neleiden, deren altes Zentrum im nördlichen Thessalien und Pierien, vor allem im alten Iolkos, dem Stammsitz der Neleiden zu suchen ist. Mit den von Neleus sich herleitenden Penthiliden gelangte diese Muse nach der Insel Lesbos und deren Pflanzstädte in der kleinasiatischen Äolis, mit den mutterländischen Neleiden nach dem zwar nur kurzlebigen aber hochberühmten Pylos. Und aus dem äolischen Kyme kam ja Hesiods Vater nach Böotien, aus dem Neleischen Pylos das Seher $=$ und Priestergeschlecht der Lykomiden nach Phlya, beide als Träger der alten Muse. War ein Kephalaion dieser Muse vom Grossen Sänger her mythisch-poetisches Gut der Demeter-PersephoneAssoziation (so nach dem Marmor Parium, das diese Orpheus-«Dichtung» im Jahre 1398 ansetzt), so war das Hauptstück der lykomidischen Gentiltradition immer noch jene gleiche der Feier ihrer Hauptgottheiten zugeordnete hymnische Orpheus-Tradition um Demeter und Persephone ${ }^{80}$.

Die konsequente Anwendung des dreiteiligen Instrumentariums in der Erklärung der neuen - bisher nur aus «Homer» bekannten - Or-

${ }^{76}$ Für die neuere Auffassung - Homer von Hesiod abhängig - ist bereits auf Bethe sowie die seinerzeit bemerkenswerte Arbeit von Fr. Schwenn verwiesen, oben A. 48. $\mathrm{Zu}$ der sonst angenommenen umgekehrten Abhängigkeit s. W. Nicolai zu Fritz Krafft, Vergleichende Unters. zu Homer und Hesiod (Hypomnemata 6, 1963), Gnomon 36, 1964, 544 ff.

7 Chantraine, REG 47, 1934, 281 ff., bes. 291. - D.L. Page, Homeric Odyssey, Oxford 1955, 36. - Hoekstra, Mnemosyne 10, 1957, 193 (dazu Année philol. 28, 1957, 84).

${ }^{78}$ So T.B.L. Webster, From Myc. to Hom., London 1958, 84 ff., 178 ff.: die gemeinsame Quelle nimmt er in "premigration poetry" an, "and therefore in all probability a Mycenaean source».

79 Orpheus als mykenische Sängergestalt, stichwortartig zusammengefasst: Peisistratos 108 «4». Dazu zuletzt Verk. Muse 152 ff. Für die nachstehende kurze Rekapitulation kann auf Verweisungen verzichtet werden.

${ }_{80}$ Beides, die "Dichtung" des Orpheus und die der Lykomiden, gehört natürlich zusammen (und zeigt die durch lange Jahrhunderte beständige Tradition, oben, $\mathbf{A}$. 14, die ja auch Webster annehmen muss, vorletzte Anm.): beides ist aber, soweit dem Vf. bekannt, noch nie zusammengebracht worden, begreiflicherweise, wenn alles was mit Orpheus zusammenhängt "spätgefälscht» und das Geschlecht der Lykomiden nur nachträglich "orphisiert» sein soll (Kl. Pauly s. Lyk.). 
pheusverse des Derveni-Papyrus erweist sich in sich selbst wie auch aus den historischen Zusammenhängen als gerechtfertigt. Mit den neu gewonnen Versen Il. V 637 + VIII 236, Hy. XV 7, Od. VIII 335, Il. XXIV $527 \mathrm{f}$. mehrt sich also der Bestand an alten Fügungen der orpheischen Muse, die durch den Letzten Dichter - den Helfer des Peisistratos und der Peisistratiden aus Lykomidengeschlecht, das sich wie das des Tyrannen vom Neleischen Pylos herleitet und aus dieser Abkunft alte musisch-poetische Orpheustradition als Gentilvermächtnis sein eigen nennt - in den von ihm «redigierten Homer» gekommen sind, Fügungen die aber natürlich auch in die aus der präliterarischen Orpheustradition geschaffenen Schriftpoesien Eingang gefunden haben.

Über die Orpheusverse im "Homer», auch die früher schon bekannten, sollte kein Zweifel mehr möglich sein. Heute noch - als blosse philologische Convention, die keiner Rechtfertigung mehr bedarf ${ }^{81}$ Lobeck zu folgen geht nicht mehr an. Die Geschichte griechischer Dichtung und musischen Wesens beginnt, wie die Alten sehr wohl wussten, mit Orpheus. Epische Probleme, namentlich die Interrelation der Grossen, Orpheus Hesiod Homer, untereinander und damit auch Fragen der epischen Chronologie, verschliessen sich einer glaubwürdigen Lösung, solange man Orpheus und das Problem der präliterarischen Orpheustradition als inexistent aus der Forschung ausschliesst.

ROBERT BOHME

${ }^{81} \mathrm{Zu}$ der den gesamten antiken Ubberlieferungen und Ưberzeugungen zuwiderlaufenden philologischen Convention hat bereits im Jahre 1954 W.K.C. Guthrie gesagt: "To fly in the face of so universal a tradition is an audacious act", Gnomon 26, 1954, 304. Doch bleibt auch danach alles beim Alten, s. Verk. Muse 144-148, daneben auch oben, A. 44 (ohne hier auf West's Buch hinsichtlich der präliterarischen Anfänge der Orpheustradition eingehen zu können). 